\title{
The Largest Liapunov Exponent for Random Matrices and Directed Polymers in a Random Environment
}

\author{
J.-P. Eckmann ${ }^{1}$ and C. E. Wayne ${ }^{2}$ \\ ${ }^{1}$ Département de Physique Théorique, Université de Genève, CH-1211 Geneva 4, Switzerland \\ ${ }^{2}$ Department of Mathematics, Pennsylvania State University, University Park, PA 16802, USA
}

\begin{abstract}
We study the largest Liapunov exponent for products of random matrices. The two classes of matrices considered are discrete, $d$-dimensional Laplacians, with random entries, and symplectic matrices that arise in the study of $d$-dimensional lattices of coupled, nonlinear oscillators. We derive bounds on this exponent for all dimensions, $d$, and we show that if $d \geqq 3$, and the randomness is not too strong, one can obtain an explicit formula for the largest exponent in the thermodynamic limit. Our method is based on an equivalence between this problem and the problem of directed polymers in a random environment.
\end{abstract}

\section{Introduction}

Consider a classical Hamiltonian system of mass points, arranged on a $d$-dimensional lattice and connected by (non-linear) springs along the lattice edges. At high energies per site, the time evolution of this system may be quite chaotic and we want to measure the chaoticity in terms of Liapunov exponents. In the case of a one dimensional lattice (i.e., a chain of oscillators) extensive numerical results $[5,8]$ suggest that the Liapunov exponents approach some sort of thermodynamic limit as the number of oscillators approaches infinity. In earlier work [3] we have argued heuristically that the Liapunov exponents for such a system could be well approximated by the Liapunov exponents of a product of random, symplectic matrices.

In the present paper we analyze rigorously the largest Liapunov exponent of a product of such matrices. We describe the exact form of those matrices below, but first we summarize our results. We first derive an upper bound for the largest Liapunov exponent of such systems, valid in all dimensions, $d$, and for any number of oscillators. We then show that for dimension $d \geqq 3$, and when the randomness is not too strong this upper bound gives the exact value of the Liapunov exponent in the thermodynamic limit. The proof consists basically of two steps. We begin by showing that one can rewrite the expression for the Liapunov exponent as a random walk in a random environment. The second step uses recent ideas of 
Imbrie and Spencer [4] to derive the desired bounds from this random walk representation.

We now describe in greater detail the class of random matrices we consider. We denote by $\mathbf{i}$ the $d$-dimensional vector

$$
\mathbf{i}=\left(i_{1}, \ldots, i_{d}\right) \text {. }
$$

We fix a rank $M$, and define

$$
\mathbf{I}_{M}=\left\{\mathbf{i}: i \leqq i_{k} \leqq M, k=1, \ldots, d\right\} .
$$

In general, we shall consider periodic boundary conditions, and thus we define

$$
|\mathbf{i}-\mathbf{j}|=\sum_{k=1}^{d} \operatorname{dist}\left(i_{k}, j_{k}\right),
$$

where the distance is measured $\bmod M^{1}$. For future use we define $\mathbf{B}_{M}$ as the set of non-directed bonds

$$
\mathbf{B}_{M}=\left\{\mathbf{b}=(\mathbf{i}, \mathbf{j}): \mathbf{i}, \mathbf{j} \in \mathbf{I}_{M},|\mathbf{i}-\mathbf{j}|=1\right\} .
$$

Now suppose we have a system of coupled oscillators whose Hamiltonian is of the form

$$
\mathscr{H}(p, q)=\sum_{\mathbf{i} \in \mathbf{I}_{M}} \frac{p_{\mathbf{i}}^{2}}{2 m}+\sum_{\substack{\mathbf{i}, \mathbf{j} \in \mathbf{I}_{M} \\|\mathbf{i}-\mathbf{j}|=1}} V\left(q_{\mathbf{i}}-q_{\mathbf{j}}\right) .
$$

If at some instant of time, say $t_{0}$, the momentum and position of the system are $(p(0), q(0))$ then for a sufficiently small interval of time $\tau$, we can approximate the trajectories of the system (1.1) by expanding the Hamiltonian in a Taylor series about $(p(0), q(0))$ and retaining only terms of second order or less. The resulting linearized equations of motion may be solved exactly, and the tangent matrix to this linearized flow is

$$
\exp \left\{\tau\left(\begin{array}{cc}
0 & \mathscr{V}(q(0)) \\
-1 & 0
\end{array}\right)\right\}
$$

with $\mathscr{V}$ the $M^{d} \times M^{d}$ matrix given by

$$
\begin{array}{ll}
\mathscr{V}(q(0))_{\mathbf{i}, \mathbf{j}}=\sum_{\mathbf{b}=(\mathbf{i}, \mathbf{k})} V^{\prime \prime}\left(q(0)_{\mathbf{i}}-q(0)_{\mathbf{k}}\right), & \text { if } \quad \mathbf{i}=\mathbf{j}, \\
\mathscr{V}(q(0))_{\mathbf{i}, \mathbf{j}}=-V^{\prime \prime}\left(q(0)_{\mathbf{i}}-q(0)_{\mathbf{j}}\right), & \text { if } \quad|\mathbf{i}-\mathbf{j}|=1,
\end{array}
$$

and all other elements are 0 .

Note that the indices of $\mathscr{V}$ are $d$ dimensional vectors. Here, and whenever we encounter such matrices in what follows, we define the product of two such matrices, $\mathscr{V}$ and $\tilde{\mathscr{V}}$ to be

$$
(\mathscr{V} \widetilde{\mathscr{V}})_{\mathbf{i}, \mathbf{j}}=\sum_{\mathbf{k}} \mathscr{V}_{\mathbf{i}, \mathbf{k}} \widetilde{\mathscr{V}}_{\mathbf{k}, \mathbf{j}}
$$

\footnotetext{
${ }^{1}$ We shall only need the notions of small distances, to describe nearest neighbors and similar things. Therefore we prefer the (incorrect but more readable) notation $|\mathbf{i}-\mathbf{j}|$ instead of $\operatorname{dist}(\mathbf{i}, \mathbf{j})$
} 
In [3] we argued that at sufficiently high energies one could choose the time interval $\tau$ such that one could simultaneously insure that the linearized approximation was valid and that at successive times $t_{0}$ and $t_{0}+\tau$, the elements of $\mathscr{V}\left(q\left(t_{0}\right)\right)$ and $\mathscr{V}\left(q\left(t_{0}+\tau\right)\right)$ would be uncorrelated with respect to one another due to the chaotic nature of the trajectories of the system. Thus, we concluded that the Liapunov exponents of the system (1.1) could be approximated by those of the product of random, symplectic, matrices

$$
S=\exp \left\{\tau\left(\begin{array}{cc}
0 & \Omega \\
-1 & 0
\end{array}\right)\right\}
$$

The matrix $\Omega$ in this equation is defined by assigning to every bond $\mathbf{b}$ in $\mathbf{B}_{M}$ (see above, for the definition of $\mathbf{B}_{M}$ ) a random variable, $\omega_{\mathbf{b}}$. These random variables are assumed to be independent and identically distributed. Note that one should think of $\omega_{\mathbf{i}, \mathbf{j}}$ as representing $\mathscr{V}_{\mathbf{i}, \mathbf{j}}$ in the above tangent matrices. Then a $M^{d} \times M^{d}$ matrix $\Omega(\omega)$ is given by

$$
\Omega_{\mathbf{i j}}=\sum_{\mathbf{b}=(\mathbf{i}, \mathbf{k})} \omega_{\mathbf{b}}, \quad \text { if } \quad \mathbf{i}=\mathbf{j}
$$

and

$$
\Omega_{\mathbf{i j}}=-\omega_{(\mathbf{i}, \mathbf{j})}, \quad \text { if } \quad|\mathbf{i}-\mathbf{j}|=1,
$$

all other elements being 0 .

In the present paper we will study rigorously the Liapunov exponents of products of random, symplectic, matrices of the form

$$
\Sigma=\left(\begin{array}{cc}
1 & \Omega \\
1 & 1+\Omega
\end{array}\right)
$$

with $\Omega$ as above. One can think of such matrices as an approximation to the $\tau=1$ case of the matrices (1.3), and although $\tau=1$ is far outside the region of applicability of the theory described above, previous numerical work $[5,8]$ (again limited to $d=1$ ) has shown that the Liapunov exponents of matrices like (1.6) behave in a fashion very similar to those of Hamiltonian systems of the type (1.1).

We now give a concise statement of our principal results. Let $\langle\ldots\rangle$ denote the average of the enclosed quantity with respect to the distribution of the random variables $\omega$. Let $\bar{\mu}$ be the largest eigenvalue of the averaged matrix $\langle\Sigma\rangle$. (Note that this eigenvalue is independent of $M$ - see Sect. 4.) We will assume that the random variables $\omega$ have a distribution with strictly positive support, together with some integrability assumptions detailed in the next section. Finally, let $\lambda_{M}$ be the largest Liapunov exponent of the product of matrices of the form $\Sigma$.

Theorem 1.1. For all dimensions $d$, and all $M$ one has

$$
\lambda_{M} \leqq \log \bar{\mu} .
$$

If $d \geqq 3$, and the variance of the random variables $\omega$ is sufficiently small, we have

$$
\lim _{M \rightarrow \infty} \lambda_{M}=\log \bar{\mu} .
$$


Note that $\log \bar{\mu}$ is the largest Liapunov exponent of the product of constant matrices $\langle\Sigma\rangle$, so we will sometimes refer to the second conclusion of Theorem 1.1 as the "self-averaging" of Liapunov exponents. For reasons discussed in Sect. 3 it is also related to the equality of the "annealed" and "quenched" averages in spin glass theory.

The only previous work with which we are familiar, that allows one to compute the largest Liapunov exponent for products of random matrices is that of Cohen and Newman [2] and Newman [6,7]. That theory requires that the distribution of the random matrices, $S$, be such that $S^{T} S$ is rotationally invariant, and that the density of states of these matrices converges to a non-random function in the thermodynamic limit. Such a condition holds, for instance, for the well known "Gaussian orthogonal ensemble" whose density of states satisfies "Wigner's circle law" in the thermodynamic limit. In [6] a formula is given which expresses the largest Liapunov exponent as one half the logarithm of the second moment of the limiting density of states. One can write down an integral equation for the density of states of the matrices $\Sigma$ in (1.6) using the methods in [3]. While it is not possible to solve that equation exactly, one can determine the support of the density of states, and one finds that the eigenvalue $\bar{\mu}$, appearing in Theorem 1.1 is precisely the upper bound of the support of the density of states, rather than the square root of its second moment. Thus we are evidently in a rather different situation than that studied by Cohen and Newman.

We conclude this introduction by reviewing the organization of the remainder of this paper. We begin in Sect. 2, by discussing a slightly simpler situation namely, the Liapunov exponents of products of the random matrices $\Omega$ introduced above. Note that in the absence of randomness, the matrices $\Omega$ would just be the $d$-dimensional lattice Laplacian, so we refer to $\Omega$ as a "random Laplacian." We introduce the random walk representation for the Liapunov exponents in this section because this allows us to introduce the principal ideas of our method in a notationally simpler context. Section 3 shows how one can bound the random walk representation for the random Laplacians, while Sect. 4 treats the additional complications encountered in the symplectic case. In Sect. 5 we give some generalizations of classical results on random walks, found for instance in [10], and finally in Sect. 6, we present numerical evidence which strongly indicates that in $d=1$, the self averaging property discussed above does not hold.

\section{Liapunov Exponents for Random Laplacians}

\subsection{Liapunov Exponents}

We are interested in computing the largest Liapunov exponent of a product of the random Laplacians $\Omega$ introduced above:

Here,

$$
\lambda_{M}=\lim _{t \rightarrow \infty} \frac{1}{t} \log \left\|\Omega^{t}(\underline{\omega}) e\right\|
$$

$$
\Omega^{t}(\underline{\omega})=\Omega\left(\omega^{(t-1)}\right) \Omega\left(\omega^{(t-2)}\right) \ldots \Omega\left(\omega^{(0)}\right),
$$

is the $t$-fold product of random matrices and $e$ is a nonzero vector. 
If we average $\Omega$-i.e., compute the matrix $\langle\Omega\rangle$ (throughout this paper, $\langle\cdot\rangle$ denotes expectation with respect to the $\omega$ 's $)$ - then $\langle\Omega\rangle$ is proportional to the $d$-dimensional lattice Laplacian, $D$, with periodic boundary conditions.

If one has a constant matrix whose largest eigenvalue is real and positive, then the largest Liapunov exponent of the product of such matrices is just the logarithm of this eigenvalue. Thus, if we compute

$$
\bar{\lambda}_{M}=\lim _{t \rightarrow \infty} \frac{1}{t} \log \left\|\langle\Omega\rangle^{t} e\right\|,
$$

this is just the logarithm of the largest eigenvalue of $D$. This eigenvalue is equal to $4 d$ (independent of $M$ ) so that $\bar{\lambda}_{M}=\log (4 d)$. In Sect. 3 we will prove upper and lower bounds relating $\lambda_{M}$ to $\bar{\lambda}_{M}$.

It is clear that the Liapunov exponent is independent of the choice of any (equivalent) norm on $\mathbf{R}^{M^{d}}$. The norm most convenient for our purposes is the $L^{1}$ norm. Throughout this paper we will take

$$
\|v\|=\sum_{\mathbf{i}}\left|v_{\mathbf{i}}\right| .
$$

\subsection{Existence of the Liapunov Exponents}

We will assume throughout that the random variables $\omega_{\mathbf{b}}^{(p)}$ have a density, $\varrho$, with

(H.1) $\operatorname{supp} \varrho \subset(0, \infty)$,

(H.2) $\log \omega \in L^{1}(\varrho(\omega) d \omega)$,

(H.3) $\omega^{2} \in L^{1}(\varrho(\omega) d \omega)$.

Definition. If a random variable $\omega$ satisfies H.1-H.3 above we shall say that $\omega \in\left(L^{0} \cap L^{2}\right)_{+}$.

Lemma 2.1 If the $\omega_{\mathbf{b}}^{(p)}$ are in $\left(L^{0}\right)_{+}$then the Liapunov exponents of the product (2.1) exist. They are almost surely independent of $\underline{\omega}$, and the largest Liapunov exponent $\lambda_{M}$ is given by

$$
\lambda_{M}=\left\langle\lim _{t \rightarrow \infty} \frac{1}{t} \log \left\|\Omega^{t}(\underline{\omega}) e\right\|\right\rangle=\lim _{t \rightarrow \infty} \frac{1}{t}\left\langle\log \left\|\Omega^{t}(\underline{\omega}) e\right\|\right\rangle .
$$

Proof. By [9], a sufficient condition for the conclusions of the lemma to hold is

$$
\left\langle\log ^{+}\|\Omega(\omega)\|\right\rangle<\infty,
$$

where $\log ^{+}(x)=\max (0, \log (x))$. We now show that (2.4) holds for our matrices $\Omega$. Since we are working with $L^{1}$ norms, and the $\omega_{\mathbf{b}}$ are all positive, we have

$$
\|\Omega\| \leqq \max _{\mathbf{j}} \sum_{\mathbf{i} \in \mathbf{I}_{M}}\left|\Omega_{\mathbf{i j}}(\omega)\right| \leqq \sum_{\mathbf{i}, \mathbf{j} \in \mathbf{I}_{M}}\left|\Omega_{\mathbf{i j}}(\omega)\right| \leqq 4 d \sum_{\mathbf{b} \in \mathbf{B}_{M}} \omega_{\mathbf{b}},
$$

where we have used the special form of $\Omega$. We next note that

$$
\begin{aligned}
\log ^{+}\|\Omega(\omega)\| & \leqq \log ^{+}\left(4 d \sum_{\mathbf{b} \in \mathbf{B}_{M}} \omega_{\mathbf{b}}\right) \leqq \log ^{+}\left(4 d M^{d} \max _{\mathbf{b} \in \mathbf{B}_{M}} \omega_{\mathbf{b}}\right) \\
& \leqq \log ^{+}\left(\max _{\mathbf{b} \in \mathbf{B}_{M}} \omega_{\mathbf{b}}\right)+\log \left(4 d M^{d}\right) .
\end{aligned}
$$


Here we first used $\operatorname{card}\left(\mathbf{B}_{M}\right)=M^{d}$. Then we used that for $x_{j}>0, \log ^{+}\left(\sum_{j=1}^{s} x_{j}\right)$
$\leqq \log \left(s \max x_{j}\right)$. But $\leqq \log \left(s \max _{j} x_{j}\right)$. But

$$
\left\langle\log ^{+}\left(\max _{\mathbf{b} \in \mathbf{B}_{M}} \omega_{\mathbf{b}}\right)\right\rangle \leqq \sum_{\mathbf{b} \in \mathbf{B}_{M}}\left\langle\log ^{+}\left(\omega_{\mathbf{b}}\right)\right\rangle<\infty
$$

because $\omega \in\left(L^{0}\right)_{+}$. Therefore (2.4) follows.

Remark. The results of [9] further imply that the limit in (2.3) is almost surely independent of the vector $e$. In fact, it does not depend on $e$ if $e$ lies outside of a given hyperplane.

\subsection{An Equivalent Problem}

Note that if the random variables $\omega_{\mathbf{b}}^{(p)}$ are identically distributed with non-zero mean, then multiplying each $\omega_{\mathbf{b}}^{(p)}$ a constant factor, $c$ just multiplies $\Omega^{t}(\underline{\omega})$ by $c^{t}$, and hence changes $\lambda_{M}$ by an additive constant $\log |c|$. Since we will assume that the random variables have non-zero mean, the above remark implies that we may assume this mean to be 1, without loss of generality. We will do so from now on.

We next remark that we can replace the matrix $\Omega(\omega)$ by one in which the off diagonal elements are positive, without changing the largest Liapunov exponent.

Lemma 2.2. Let $\Omega(\omega)$ be a family of random matrices as defined in Eqs. (1.4), (1.5), and assume that all $\omega_{\mathbf{i}}^{(p)}$ have the same sign. If $M$ is even, then the largest Liapunov exponent of $\Omega(\omega)$ coincides with the largest Liapunov exponent of $\Gamma(\omega)$, where

$$
\Gamma(\omega)_{\mathbf{i j}}=\left\{\begin{aligned}
\Omega_{\mathbf{i} \mathbf{j}}, & \text { if } \mathbf{i}=\mathbf{j}, \\
-\Omega_{\mathbf{i j}}, & \text { otherwise. }
\end{aligned}\right.
$$

Proof. This is similar to the relation between ferromagnets and antiferromagnets. Choose an initial vector $e^{P}$ with the property that

$$
e_{\mathbf{i}}^{P}=(-1)^{P(\mathbf{i})} e_{\mathbf{i}},
$$

where the "parity" $P$ is defined by

$$
P(\mathbf{i})=\sum_{k=1}^{d} i_{k}
$$

and $e_{\mathbf{i}} \geqq 0$. In the sequel, $\underline{\mathbf{i}}$ denotes $(t+1)$-tuples $\left(\mathbf{i}^{(0)}, \ldots, \mathbf{i}^{(t)}\right)$, with $\mathbf{i}^{(p)} \in \mathbf{I}_{M}$. It is then easy to see that all terms in the sum below have the same sign:

$$
\left(\Omega^{t}(\underline{\omega}) e^{P}\right)_{\mathbf{j}}=\sum_{\underline{i}} \Omega_{\mathbf{j} \mathbf{i}^{(t-1)}}\left(\omega^{(t-1)}\right) \Omega_{\mathbf{i}^{(t-1)} \mathbf{i}^{(t-2)}}\left(\omega^{(t-2)}\right) \ldots \Omega_{\mathbf{i}^{(1)} \mathbf{i}^{(0)}}\left(\omega^{(0)}\right) e_{\mathbf{i}^{(0)}}^{P} .
$$

In fact, this sign is even independent of $\mathbf{j}$. Taking absolute values, we see that

$$
\left|\left(\Omega^{t}(\underline{\omega}) e^{P}\right)_{\mathbf{j}}\right|=\left|\left(\Gamma^{t}(\underline{\omega}) \tilde{e}\right)_{\mathbf{j}}\right|
$$

where $\tilde{e}_{\mathbf{i}}=e_{\mathbf{i}}$ for all $\mathbf{i} \in \mathbf{I}_{M}$. Thus,

$$
\lim _{t \rightarrow \infty} \frac{1}{t} \log \left\|\Omega^{t}(\underline{\omega}) e^{P}\right\|=\lim _{t \rightarrow \infty} \frac{1}{t} \log \left\|\Gamma^{t}(\underline{\omega}) \tilde{e}\right\| .
$$

Since this holds for all $\underline{\omega}$ and for $e^{P}$ and $\tilde{e}$ in a set of positive measure, it implies that the Liapunov exponents are equal. 


\subsection{The Random Walk Expansion}

The main point of this section is an interpretation of $\left\|\Gamma^{t}(\underline{\omega}) e\right\|$ as a random walk. We will assume that the components of $e$ are non-negative. Then

$$
\begin{aligned}
\left\|\Gamma^{t}(\underline{\omega}) e\right\| & =\sum_{\mathbf{i} \in \mathbf{I}_{M}}\left|\left(\Gamma^{t}(\underline{\omega}) e\right)_{\mathbf{i}}\right|=\sum_{\mathbf{i} \in \mathbf{I}_{M}}\left(\Gamma^{t}(\underline{\omega}) e\right)_{\mathbf{i}} \\
& \left.=\sum_{\mathbf{i}, \mathbf{j} \in \mathbf{I}_{M}}\left(\sum_{\left.\mathbf{i}: \mathbf{i}^{(t)}\right)} \sum_{\mathbf{i}, \mathbf{i}^{(0)}} \prod_{p=0}^{t-1} \Gamma_{\mathbf{i}^{(p+1)}}\right)_{\mathbf{i}(p)}\left(\omega^{(p)}\right) e_{\mathbf{j}}\right) .
\end{aligned}
$$

Note in particular if we choose the initial vector, $e$, to have component $e_{\mathbf{i}_{0}}=1$, and all others zero, where $\mathbf{i}_{0}$ is an arbitrary element of $\mathbf{I}_{M}$, then we have

$$
\left\|\Gamma^{t}(\underline{\omega}) e\right\|=\sum_{\mathbf{i}: \mathbf{i}^{(0)}=\mathbf{i}_{0}} \prod_{p=0}^{t-1} \Gamma_{\mathbf{i}^{(p+1)} \mathbf{i}_{\mathbf{i}}(p)}\left(\omega^{(p)}\right) .
$$

Note 2.3. The proofs we are giving are independent of the particular choice of $\mathbf{i}_{0}$ and any other vector with positive components would be acceptable. Throughout, we shall fix $\mathbf{i}_{0}$. As we remarked earlier in this section, the largest Liapunov exponent is independent of the initial vector so long as it lies outside of some hyperplane. Since our results are independent of which of the $M^{d}$ vectors $e_{i_{0}}$ that we choose, they must give the largest Liapunov exponent.

We interpret $\mathbf{i}$ as a random walk in $\mathbf{I}_{M}$, where at time $p$ we step from $\mathbf{i}^{(p)}$ to $\mathbf{i}^{(p+1)}$. Because of the sparseness of $\Omega$ and $\Gamma$, the only nonzero terms in (2.5) are those in which only "horizontal" and "diagonal" steps occur, corresponding, respectively to $\mathbf{i}^{(p)}=\mathbf{i}^{(p+1)}$ and $\left|\mathbf{i}^{(p)}-\mathbf{i}^{(p+1)}\right|=1$.

We associate to each walk a probability $w_{t}^{(M)}(\mathbf{i})$ and a weight, which is one in the absence of randomness. The probability density $w_{t}^{(M)}(\mathbf{i})$ comes about as follows. We have a representation similar to (2.5) for $\left\|\langle\Gamma\rangle^{t} e\right\|$, and the contribution in this case is a factor of $2 d$ for every "horizontal" step and a factor of 1 for every "diagonal" step. This makes $\mathbf{I}_{M}{ }^{t}$ a probability space with weight $w_{t}^{(M)}(\mathbf{i})$, when we normalize properly. We call these walks "free" walks.

More precisely, let $f_{0}=2 d$ and $f_{1}=1$, and $f_{j}=0$ for $j \geqq 2$, and define

and finally

$$
\dot{w}_{t}^{(M)}(\mathbf{i})=\prod_{p=0}^{t-1} f_{\mid \mathbf{i}^{(p+1)}-\mathbf{i}^{(p) \mid}}
$$

$$
w_{t}^{(M)}(\mathbf{i})=\frac{\dot{w}_{t}^{(M)}(\mathbf{i})}{\sum_{\mathbf{j}: \mathbf{j}^{(0)}=\mathbf{i}_{0}} \grave{w}_{t}^{(M)}(\mathbf{j})} .
$$

It should be noted that, in principle, the normalization factor depends on $\mathbf{i}_{0}$. In the case of the random Laplacian, it is in fact independent of $\mathbf{i}_{0}$, and equal to $(4 d)^{t}$. We shall, however, not use this information, so that the generalization of our methods to the case of random symplectic matrices will be easier. It will also be useful to define

so that

$$
\dot{\mathfrak{w}}_{t_{1}, t_{2}}(\mathbf{i})=\prod_{p=t_{1}}^{t_{2}-1} f_{|\mathbf{i}(p+1)-\mathbf{i}(p)|},
$$

$$
\stackrel{\circ}{w}_{t}^{(M)}(\mathbf{i})=\stackrel{\circ}{w}_{0, t}(\mathbf{i}) \text {. }
$$


Definition of the Variables $\sigma$. We now introduce new random variables, $\sigma$, defined by $\sigma_{\mathbf{b}}=\omega_{\mathbf{b}}-1$ if the bond $\mathbf{b}$ is diagonal, and $\sigma_{\mathbf{i} \mathbf{i}}=\sum_{\mathbf{j}:|\mathbf{j}-\mathbf{i}|=1}\left(\omega_{\mathbf{i} \mathbf{j}}-1\right) / 2 \mathrm{~d}$, for horizontal bonds. In more generic notation, we can write for $\mathbf{b}=(\mathbf{i}, \mathbf{j})$,

whenever $|\mathbf{i}-\mathbf{j}| \leqq 1$.

$$
\sigma_{\mathbf{i j}}=\frac{\Gamma_{\mathbf{i j}}-\left\langle\Gamma_{\mathbf{i j}}\right\rangle}{\left\langle\Gamma_{\mathbf{i j}}\right\rangle},
$$

Remarks. 1) With the above definition, all $\sigma_{\mathbf{b}}$ have mean zero.

2) By hypothesis H.2, and because $\varrho$ is a probability measure, the $\sigma_{\mathbf{b}}$ have finite variance. We define

$$
\sigma^{2}=\max _{\mathbf{b}}\left(\operatorname{var}\left(\sigma_{\mathbf{b}}\right)\right)
$$

In terms of $\sigma$ we get the following nice representation for $\lambda_{M}-\bar{\lambda}_{M}$ :

$$
\lambda_{M}-\bar{\lambda}_{M}=\lim _{t \rightarrow \infty} \frac{1}{t} \log \frac{\left\|\Gamma^{t}(\underline{\omega}) e\right\|}{\left\|\langle\Gamma\rangle^{t} e\right\|} \equiv \lim _{t \rightarrow \infty} \frac{1}{t} \log Z_{t},
$$

with the identities

and

$$
\grave{Z}_{t}^{(M)}(\underline{\sigma})=\sum_{\mathbf{i}: \mathbf{i}^{(0)}=\mathbf{i}_{0}} \grave{w}_{t}^{(M)}(\mathbf{i}) \prod_{p=0}^{t-1}\left(1+\sigma_{\mathbf{i}(p)}^{(p)} \mathbf{i}^{(p+1)}\right),
$$

$$
\begin{aligned}
& Z_{t}^{(M)}(\underline{\sigma})=\frac{\stackrel{Z}{t}_{t}^{(M)}(\underline{\sigma})}{\left\langle\dot{Z}_{t}^{(M)}(\underline{\sigma})\right\rangle}=\frac{\sum_{\mathbf{i}: \mathbf{i}^{(0)}=\mathbf{i}_{0}} \stackrel{\circ}{t}_{t}^{(M)}(\underline{\mathbf{i}}) \prod_{p=0}^{t-1}\left(1+\sigma_{\mathbf{i}(p)}^{(p)} \mathbf{i}^{(p+1)}\right)}{\sum_{\mathbf{i}: \mathbf{i}^{(0)}=\mathbf{i}_{0}} \dot{w}_{t}^{(M)}(\mathbf{i})} \\
& =\sum_{\mathbf{i}: \mathbf{i}^{(0)}=\mathbf{i}_{0}} w_{t}^{(M)}(\underline{\mathbf{i}}) \prod_{p=0}^{t-1}\left(1+\sigma_{\mathbf{i}(p)}^{(p)} \mathbf{i}^{(p+1)}\right) .
\end{aligned}
$$

Notation. We shall use henceforth the more readable notation

$$
\sigma(p ; \underline{\mathbf{i}})=\sigma_{\mathbf{i}(p)}^{(p)} \mathbf{i}_{(p+1)} .
$$

Note that $Z_{t}(\underline{\sigma})$ is precisely the partition function for the diffusion of directed polymers in a random environment, [4]. In general, we will not write explicitly the $M$-dependence of $Z_{t}(\sigma)$, but it is understood that the random paths vary in $\mathbf{I}_{M}$. In this diffusion the random paths are chosen with a probability which is independent of the random environment $\underline{\sigma}$. We are diffusing here on a finite lattice with periodic boundary conditions (of period $M, M$ even), and hence the results of Imbrie and Spencer are not directly relevant for our questions. We will see in Sect. 3 how some of their ideas can be made applicable to our case.

\subsection{Inequalities for Arbitrary Dimension}

Using the random walk expansion of Sect. 2.4, we may easily derive some rigorous bounds on the largest Liapunov exponent, cf. also [1] for other bounds.

Proposition 2.4. If the random variables $\omega_{\mathbf{i}}$ are in $\left(L^{0}\right)_{+}$, i.e., if they satisfy

$$
\int d \mu(\omega)|\log (|\omega|)|<\infty,
$$


then for all even $M$ and in all dimensions $d \geqq 1$ one has the inequality

$$
\lambda_{M} \leqq \bar{\lambda}_{M} .
$$

Proof. Since $\lambda_{M}$ is almost surely independent of $\underline{\omega}$ (and hence $\underline{\sigma}$ ), we may average over $\underline{\omega}$ (respectively $\underline{\sigma}$ ) and obtain

$$
\lambda_{M}-\bar{\lambda}_{M}=\left\langle\lim _{t \rightarrow \infty} \frac{1}{t} \log Z_{t}(\underline{\sigma})\right\rangle .
$$

We now note the important fact that all the random variables $\sigma_{i j}^{(p)}$ have mean zero and are independent, identically distributed for different $p$. (The distribution is also independent of $\mathbf{i}$ and $\mathbf{j}$ in the sense that there is one distribution for the case $\mathbf{i}=\mathbf{j}$ and another for the case $|\mathbf{i}-\mathbf{j}|=1$, cf. the definition of $\sigma$.)

Definition. We summarize these properties as the "time-independence" of the $\sigma$ 's.

By the time-independence of the $\sigma$ 's, we have the identity

$$
\left\langle Z_{t}(\underline{\sigma})\right\rangle=\sum_{\underline{i}: \mathbf{i}^{(0)}=\mathbf{i}_{0}} w_{t}^{(M)}(\underline{\mathbf{i}}) \prod_{p=0}^{t-1}\langle 1+\sigma(p ; \underline{\mathbf{i}})\rangle=\sum_{\mathbf{i}: \mathbf{i}^{(0)}=\mathbf{i}_{0}} w_{t}^{(M)}(\underline{\mathbf{i}})=1 .
$$

By (2.3), the interchange of the limit and the average in (2.14) is allowed and we find, by Jensen's inequality,

$$
\lambda_{M}-\bar{\lambda}_{M}=\lim _{t \rightarrow \infty} \frac{1}{t}\left\langle\log Z_{t}(\sigma)\right\rangle \leqq \lim _{t \rightarrow \infty} \frac{1}{t} \log \left\langle Z_{t}(\sigma)\right\rangle=0,
$$

and the claim follows.

This proposition may be easily generalized to yield

Lemma 2.5. Given a probability measure @ on a set of matrices $\{S(\omega)\}$ such that $\log \|S(\cdot)\| \in L^{1}(\varrho d \omega)$, define

$$
S^{\mathrm{av}}=\left\langle\left(\begin{array}{cccc}
\left|s_{11}\right| & \left|s_{12}\right| & \ldots & \left|s_{1 m}\right| \\
\vdots & & & \vdots \\
\left|s_{m 1}\right| & \left|s_{m 2}\right| & \ldots & \left|s_{m m}\right|
\end{array}\right)\right\rangle,
$$

i.e., we replace each element by the mean of its absolute value. Let $\bar{\mu}$ be the largest egienvalue of $S^{\mathrm{av}}$. Then the Liapunov exponent, $\lambda$, of the product of such matrices satisfies

$$
\lambda \leqq \log |\bar{\mu}| \text {. }
$$

Proof. Since $\lambda$ is almost surely independent of $\omega$, we have

$$
\lambda=\langle\lambda\rangle=\lim _{t \rightarrow \infty} \frac{1}{t}\left\langle\log \left\|S^{t} e\right\|\right\rangle .
$$

Here, the interchange of the limit and the average is justified as in the proof of Proposition 2.4. By Jensen's inequality, the right-hand side of (2.16) is bounded by

$$
\lim _{t \rightarrow \infty} \frac{1}{t} \log \left\langle\left\|S^{t} e\right\|\right\rangle \text {. }
$$

But $\left\langle\left\|S^{t} e\right\|\right\rangle \leqq\left\|\left(S^{\mathrm{av}}\right)^{t} e\right\| \leqq|\bar{\mu}|^{t}\|e\|$, and the lemma follows. 
We remark that in the case where the random variables are almost surely nonnegative, Proposition 2.4 will in general give sharper estimates than the bounds of [1], which are based on the $L^{2}$ norm.

Jensen's inequality also leads to a lower bound on $\lambda_{M}$. For this bound we observe that

$$
\begin{aligned}
\lambda_{M}-\bar{\lambda}_{M} & =\lim _{t \rightarrow \infty} \frac{1}{t}\left\langle\log Z_{M}(\underline{\sigma})\right\rangle \\
& =\lim _{t \rightarrow \infty} \frac{1}{t}\left\langle\log \left\{\sum_{\mathbf{i}: \mathbf{i}(0)=\mathbf{i}_{0}} w_{t}^{(M)}(\mathbf{i}) \prod_{p=0}^{t-1}(1+\sigma(p ; \underline{\mathbf{i}}))\right\}\right\rangle \\
& \geqq \lim _{t \rightarrow \infty} \frac{1}{t} \sum_{\mathbf{i}: \mathbf{i}(\tilde{\sigma})=\mathbf{i}_{0}} w_{t}^{(M)}(\mathbf{i})\left\langle\log \prod_{p=0}^{t-1}(1+\sigma(p ; \underline{\mathbf{i}}))\right\rangle .
\end{aligned}
$$

This step used the fact that $w_{t}^{(M)}(\mathbf{i})$ is the density of a probability measure. Note next that

$$
\langle\log (1+\sigma(p ; \mathbf{i}))\rangle \geqq \min \left(\left\langle\log \left(1+\sigma_{\mathbf{i i}}^{(p)}\right)\right\rangle,\left\langle\log \left(1+\sigma_{\mathbf{i j}}^{(p)}\right)\right\rangle\right) \equiv C_{\sigma},
$$

where in the second average $|\mathbf{i}-\mathbf{j}|=1$. Thus, since the distribution of $\log (1+\sigma(p ; \mathbf{i}))$ is independent of $p,(2.17)$ is bounded below by $C_{\sigma}$, and we have the

Proposition 2.6. For all $M$ and all $d \geqq 1$, the inequality

$$
\lambda_{M}-\bar{\lambda}_{M} \geqq C_{\sigma}
$$

holds. The constant $C_{\sigma}$ tends to zero if the $\omega$ tend to one in $\left(L^{0}\right)_{+}$.

\section{Three or More Dimensions}

We have already derived upper and lower bounds on the largest Liapunov exponent valid in all dimensions $d$. In the present section we show that for dimension $d \geqq 3$, one can compute the largest Liapunov exponent explicitly in the thermodynamic limit, i.e., when $M \rightarrow \infty$. Our main result is

Theorem 3.1. If $d \geqq 3$ and the variance $\sigma^{2}$ of the noise is sufficiently small then

$$
\lim _{M \rightarrow \infty} \lambda_{M}=\log (4 d) \text {. }
$$

In thermodynamic language, this says that the quenched average of $\log Z_{M}$ is equal to the annealed average. Note that the right-hand side of this equality is just $\bar{\lambda}_{M}$, (which is independent of $M !$ ) and we will prove the theorem by showing that

$$
\lim _{M \rightarrow \infty}\left(\lambda_{M}-\bar{\lambda}_{M}\right)=0 \text {. }
$$

To establish (3.1) it is only necessary to prove a lower bound on $\lambda_{M}-\bar{\lambda}_{M}$ since Proposition 2.4 implies $\lambda_{M}-\bar{\lambda}_{M} \leqq 0$ for all $M$. From the random walk expansion of Sect. 2.4 we know that

$$
\lambda_{M}-\bar{\lambda}_{M}=\lim _{t \rightarrow \infty} \frac{1}{t}\left\langle\log Z_{t}^{(M)}(\underline{g})\right\rangle,
$$


and we shall prove the lower bound by using this representation. We indicate the $M$-dependence explicitly only when it seems necessary, cf. (2.11), (2.12).

Before entering into the technical details that follow, we review their goal. We wish to use the ideas of Imbrie and Spencer [4] to show that fluctuations of $Z_{t}(\sigma)$ about its mean value of 1 are small. The method of [4] is based on the fact that in $d \geqq 3$, a random walk in a nonrandom environment is non-recurrent. However, since the walks that define the partition function, $Z_{t}(\sigma)$, in our problem take place on the finite lattice $\mathbf{I}_{M}$, they will always be recurrent after a sufficiently long time [in fact, $\left.\mathcal{O}\left(M^{d}\right)\right]$. In the following paragraphs we show that we can estimate the partition function by walks of sufficiently short length so that they do not "feel" the finite size of the lattice, and this allows us to use some of the methods of Imbrie and Spencer.

\subsection{Reduction to the Infinite Volume Case}

Throughout this section, $M$ is a fixed, even, and finite number, all walks are in $\mathbf{I}_{M}$, and the $\sigma$ are random variables indexed by time and by bonds in $\mathbf{B}_{M}$.

We may evaluate the limit of (3.2) along any convenient subsequence and we choose $\left\{t_{l}\right\}=l L$, where $L$ is a large integer. We will always choose $L<M / 2$. We will provide a lower bound by "decoupling" the partition function over "time" and then giving lower bounds on the decoupled factors. We begin by:

Proposition 3.2. There is a constant $C$ such that for any $L$, and for any dimension $d$, we have

$$
\frac{1}{l L}\left\langle\log \check{Z}_{l L}(\underline{\sigma})\right\rangle \geqq \frac{1}{L}\left\langle\log \check{Z}_{L}(\sigma)\right\rangle-\frac{C}{L} \log L,
$$

for $l=1,2, \ldots$ In particular,

$$
\lambda_{M}-\bar{\lambda}_{M} \geqq \frac{1}{L}\left\langle\log Z_{L}(\underline{\sigma})\right\rangle-\frac{C}{L}(\log L+\log M) .
$$

Proof. To prove Proposition 3.2, we note that since $Z_{l L}$ is defined as a sum over random walks, all of which have positive weights, we obtain lower bounds if we exclude some of the allowed walks. We now describe these exclusions in detail.

We define a new class of partition functions: For $t>s$, we set

$$
Z_{\mathbf{y}, \mathbf{z}}^{*}(s, t ; \underline{\sigma})=\sum_{\mathbf{i} \in \mathbf{I}_{M^{t-s+1}}}^{\sum^{*}} \dot{\circ}_{s, t}(\mathbf{i}) \prod_{p=s}^{t-1}(1+\sigma(p ; \underline{\mathbf{i}})),
$$

where the * on the sum denotes a restriction of the sum to walks $\mathbf{i}$ which "walk" only from time $p=s$ to time $p=t$, and satisfy the boundary conditions $\mathbf{i}^{(s)}=\mathbf{y}, \mathbf{i}^{(t)}=\mathbf{z}$. Note that the $\stackrel{w}{*}$ are unnormalized weights.

We fix the noise configuration, $\underline{\sigma}$, and define the recursively a sequence of "stopping points" $\left\{\mathbf{y}_{j}(\underline{\sigma})\right\}_{j=0}^{l}$, for $Z_{t_{l}}(\underline{\sigma})$ as follows:

Definition 3.3. We set $\mathbf{y}_{0}(\underline{\sigma})=\mathbf{i}_{0}$, and, for $j=0, \ldots, l-1$, we define $\mathbf{y}_{j+1}(\underline{\sigma})$ as a point y which maximizes

$$
Z_{\mathbf{y}_{j}(\sigma), \mathbf{y}}^{*}\left(t_{j}, t_{j+1} ; \underline{\sigma}\right)
$$

with $t_{j}=j L$. (One can choose any maximizing $\mathbf{y}$.) 
Note that with this definition, one has

$$
\grave{Z}_{t_{l}}(\sigma) \geqq \prod_{j=0}^{l-1} Z_{\mathbf{y}_{j}(\underline{\sigma}), \mathbf{y}_{j+1}(\underline{\sigma})}^{*}\left(t_{j}, t_{j+1} ; \underline{\sigma}\right),
$$

because the right-hand side corresponds to a restriction of paths. Thus,

$$
\frac{1}{t_{l}}\left\langle\log Z_{t_{l}}(\sigma)\right\rangle \geqq \frac{1}{t_{l}} \sum_{j=0}^{l-1}\left\langle\log Z_{\mathbf{y}_{j}(\sigma), \mathbf{y}_{j+1}(\sigma)}^{*}\left(t_{j}, t_{j+1} ; \sigma\right)\right\rangle .
$$

We compute the terms on the right-hand side of (3.6) by conditioning on the event that $\mathbf{y}_{j}(\underline{\sigma})=\mathbf{y}$. Thus,

$$
\begin{aligned}
& \left\langle\log Z_{\mathbf{y}_{j}(\sigma), \mathbf{y}_{j+1}(\sigma)}^{*}\left(t_{j}, t_{j+1} ; \sigma\right)\right\rangle \\
& \quad=\sum_{\mathbf{y} \in \mathbf{I}_{M}}\left\langle\log Z_{\mathbf{y}, \mathbf{y}_{j+1}(\sigma)}^{*}\left(t_{j}, t_{j+1} ; \sigma\right) \mid \mathbf{y}_{j}(\sigma)=\mathbf{y}\right\rangle \operatorname{Prob}\left(\left\{\underline{\sigma} \mid \mathbf{y}_{j}(\sigma)=\mathbf{y}\right\}\right) .
\end{aligned}
$$

There are now two important observations. First, note that $Z_{\mathbf{y}, \mathbf{y}_{j+1}(\sigma)}^{*}\left(t_{j}, t_{j+1} ; \underline{\sigma}\right)$ depends only on the random variables $\sigma_{\mathbf{i j}}^{(p)}$ with $t_{j} \leqq p<t_{j+1}$. In particular, by timeindependence, the random variables $Z_{\mathbf{y}, \mathbf{y}_{j+1}(\underline{\sigma})}^{*}\left(t_{j}, t_{j+1} ; \underline{\sigma}\right)$ will have the same distribution as $Z_{\mathbf{y}, \mathbf{y}_{m+1}(\sigma)}^{*}\left(t_{m}, t_{m+1} ; \underline{\sigma}\right)$ (with a relabeling of random variables). The second important observation is that because of our periodic boundary conditions,

$$
\text { the distribution of } Z_{\mathbf{y}, \mathbf{y}_{j+1}(\sigma)}^{*}\left(t_{j}, t_{j+1} ; \sigma\right) \text { is independent of } \mathbf{y} \text {. }
$$

These two observations imply that

$$
\sum_{j=0}^{l-1}\left\langle\log Z_{\mathbf{y}_{j}(\sigma), \mathbf{y}_{j+1}(\sigma)}^{*}\left(t_{j}, t_{j+1} ; \sigma\right)\right\rangle=l\left\langle\log Z_{\mathbf{y}_{0}, \mathbf{y}_{1}(\sigma)}^{*}(0, L ; \sigma)\right\rangle .
$$

Thus we get the bound

$$
\frac{1}{t_{l}}\left\langle\log \grave{Z}_{t_{l}}(\sigma)\right\rangle \geqq \frac{1}{L}\left\langle\log Z_{\mathbf{y}_{0}, \mathbf{y}_{1}(\sigma)}^{*}(0, L ; \sigma)\right\rangle .
$$

Note next that

$$
\check{Z}_{L}(\underline{\sigma})=\sum_{\mathbf{y} \in \mathbf{I}_{M}} Z_{\mathbf{y}_{0}, \mathbf{y}}^{*}(0, L ; \underline{\sigma})
$$

Since $Z_{\mathbf{y}_{0}, \mathbf{y}}^{*}(0, L ; \underline{\sigma})=0$ if $\left|\mathbf{y}-\mathbf{y}_{0}\right|>L$, (each step of the walk has length at most $\left.1 !\right)$ we have at most $\mathcal{O}\left(L^{d}\right)$ terms in the sum (3.9). Furthermore, $Z_{\mathbf{y}_{0}, \mathbf{y}_{1}(\underline{\sigma})}^{*}(0, L ; \sigma)$ is the largest term in this sum, and therefore

$$
Z_{\mathbf{y}_{0}, \mathbf{y}_{1}(\sigma)}^{*}(0, L ; \sigma) \geqq \frac{C}{L^{d}} \grave{Z}_{L}(\underline{\sigma})
$$

This proves (3.3). We now turn to the evaluation of $\left\langle Z_{t_{l}}(\sigma)\right\rangle$. We prefer to do this in a slightly more complicated fashion than is absolutely necessary, because this will allow a more straightforward generalization to the symplectic case. Note that

$$
\left\langle\dot{Z}_{t_{l}}(\sigma)\right\rangle=\left\|\langle\Gamma\rangle^{t_{l}} e\right\| \text {. }
$$

Now $\langle\Gamma\rangle$ is a fixed matrix whose largest eigenvalue, $\mu_{1}$, is simple. If $e$ has a nonzero component in the direction of this largest eigenvalue, and if $\mu_{2}$ is a bound on 
the remainder of the spectrum, $0<\mu_{2}<\mu_{1}$, then we have

$$
\left\|\langle\Gamma\rangle^{t_{l}} e\right\|=\mathrm{const} \mu_{1}^{t_{l}} \cdot\left(1+\mathcal{O}\left(M\left(\frac{\mu_{2}}{\mu_{1}}\right)^{t_{l}}\right)\right) \text {. }
$$

Thus, for all $l$,

$$
\left|\frac{1}{t_{l}} \log \left\langle{\stackrel{\circ}{t_{l}}}_{l}(\underline{\sigma})\right\rangle-\frac{1}{L} \log \left\langle\stackrel{\circ}{Z}_{L}(\underline{\sigma})\right\rangle\right| \leqq \mathcal{O}\left(\frac{\log M}{L}\right) .
$$

Combining (3.3) with (3.11), we have

$$
\begin{aligned}
\lambda_{M}-\bar{\lambda}_{M} & =\lim _{l \rightarrow \infty} \frac{1}{t_{l}}\left(\left\langle\log \dot{Z}_{t_{l}}^{(M)}(\underline{\sigma})\right\rangle-\log \left\langle\dot{Z}_{t_{l}}^{(M)}(\underline{\sigma})\right\rangle\right) \\
& \geqq \frac{1}{L}\left(\left\langle\log \dot{Z}_{L}^{(M)}(\underline{\sigma})\right\rangle-\log \left\langle\dot{Z}_{L}^{(M)}(\underline{\sigma})\right\rangle\right)-\mathcal{O}\left(\frac{\log L+\log M}{L}\right) \\
& =\frac{1}{L}\left\langle\log Z_{L}^{(M)}(\underline{\sigma})\right\rangle-\mathcal{O}\left(\frac{\log L+\log M}{L}\right) .
\end{aligned}
$$

This completes the proof of Proposition 3.2.

Remark 3.4. Had we used the explicit form of $\langle\Omega\rangle$, the term containing $\log M$ would be absent in the above estimate.

We now compare the partition function $Z_{L}^{(M)}\left(\underline{\sigma}^{(M)}\right)$ to $Z_{L}^{(\infty)}\left(\underline{\sigma}^{(\infty)}\right)$. For this we need to compare configurations of $\sigma$ 's on $\mathbf{I}_{M}$ and on $\mathbf{Z}^{d}=\mathbf{I}_{\infty}$.

Definition 3.5. We say that $\underline{\sigma}^{(M)}$ coincides with $\underline{\sigma}^{(\infty)}$ in a disk of radius $L<M / 2$ around $\mathbf{i}_{0}$ if

$$
\sigma_{\mathbf{i j}}^{(p)}=\sigma_{\mathbf{i j}}^{\prime(p)},
$$

for $p=0,1, \ldots$, and $\left|\mathbf{i}-\mathbf{i}_{0}\right| \leqq L,\left|\mathbf{j}-\mathbf{i}_{0}\right| \leqq L$ (measured on $\mathbf{I}_{M}$ for $\underline{\sigma}$ and on $\mathbf{I}_{\infty}$ for $\underline{\sigma}^{\prime}$ ).

In other words, we first extend the configuration $\sigma$ periodically from $\mathbf{I}_{M}$ to $\mathbf{Z}^{d}$. It is the configuration which is then compared to $\sigma^{\prime}$ on a disk centered at $\mathbf{i}_{0}$.

Proposition 3.6. If $M$ is even and $L<M / 2$ and $\underline{\sigma}^{(M)}$ coincides with $\underline{\sigma}^{(\infty)}$ in a disk of radius $L<M / 2$ around $\mathbf{i}_{0}$ one has

$$
Z_{L}^{(M)}\left(\underline{\sigma}^{(M)}\right)=Z_{L}^{(\infty)}\left(\underline{\sigma}^{(\infty)}\right)
$$

Proof. We have already pointed out that in a "time," $L$, the walk can move a distance at most $L$ from its initial point $\mathbf{i}_{0}$. Since $L<M / 2$, no two walks in the sum defining $Z_{L}^{(M)}(\underline{\sigma})$ can ever reach points a distance $M$, or more, from one another. Therefore we will not change the distribution of the random variable $Z_{L}^{(M)}(\underline{\sigma})$ if we replace the periodic array of random variables on $\mathbf{Z}^{d}$ (outside a disk of radius $L$ around $\mathbf{i}_{0}$ ) with a set of independent random variables with the same distribution. This completes the proof of Proposition 3.6.

\subsection{The Infinite Volume Case}

By Proposition 3.6 we see that it suffices to control the limit, as $L$ goes to $\infty$ of

$$
\frac{1}{L} \log \left\langle Z_{L}^{(\infty)}\left(\underline{\sigma}^{(\infty)}\right)\right\rangle
$$


In this subsection, $M$ is always infinite, the random walks are on $\mathbf{Z}^{d}$ starting at $\mathbf{i}_{0}$, and the weights are $w_{t}^{(\infty)}(\mathbf{i})$. This means that we are in a situation very similar to the one considered by Imbrie and Spencer. We henceforth omit the superscript $(\infty)$.

We first use the assumption H.1 we made on the random variables $\omega_{\mathbf{i j}}$ (or equivalently $\sigma_{\mathbf{i j}}$ ), in the following form:

Hypothesis. The support of the random variables $\omega_{\mathbf{i j}}$ is contained in the interval $\left[e^{-\mu}, \infty\right)$ for some $\mu, 0<\mu<\infty$.

This hypothesis gives an (obvious) pointwise lower bound on the partition function which is of the form

$$
Z_{L}(\underline{\sigma}) \geqq e^{-\mu L} .
$$

Before starting the Imbrie-Spencer part of the argument we make one more simplification. Define, for $x>0, \quad \log ^{-}(x)=\max (0,-\log (x))$ and $\log ^{+}(x)$ $=\max (0, \log (x))$. With this notation,

$$
\log Z_{L}=\log ^{+}\left(Z_{L}\right)-\log ^{-}\left(Z_{L}\right)
$$

Since we only need to derive a lower bound on $(1 / L) \log Z_{L}$, we can replace $\log Z_{L}$ by $-\log ^{-}\left(Z_{L}\right)$, and we have

$$
\frac{1}{L}\left\langle\log Z_{L}(\sigma)\right\rangle \geqq-\frac{1}{L}\left\langle\log ^{-}\left(Z_{L}(\sigma)\right)\right\rangle .
$$

We also note that (3.13) implies

$$
0 \leqq \frac{1}{L} \log ^{-}\left(Z_{L}(\sigma)\right) \leqq \mu
$$

so that we have an a priori uniform bound.

Proposition 3.7. If the noise is sufficiently weak, and $d \geqq 3$, then we have, for $L_{j}=2^{j}$,

$$
\lim _{j \rightarrow \infty} \frac{1}{L}\left\langle\log ^{-}\left(Z_{L_{j}}(\sigma)\right)\right\rangle=0 \text {. }
$$

Proof of Theorem 3.1. If we combine (3.4) and (3.14) with (3.16), then we see that for every $\varepsilon>0$ and $M$ sufficiently large,

$$
\lambda_{M}-\bar{\lambda}_{M}>-\varepsilon .
$$

Since we have already shown $\lambda_{M}-\bar{\lambda}_{M} \leqq 0$, this completes the proof of Theorem 3.1 .

The remainder of this section is essentially devoted to the proof of Proposition 3.7. We need first a notion of "irreducible part" of the partition function. We begin by defining, for $s<t$,

$$
p_{I}\left(t, \mathbf{i}_{t} ; s\right)=\sum_{\mathbf{i}: \mathbf{i}^{(s)}=\mathbf{i}_{0}, \mathbf{i}^{(t)}=\mathbf{i}_{t}} \sigma(t-1 ; \mathbf{i}) w_{s, t}(\underline{\mathbf{i}}) \prod_{p=s}^{t-2}(1+\sigma(p ; \underline{\mathbf{i}})) .
$$

(Recall that $w$ is the (normalized) probability on the infinite domain $\mathbf{Z}^{d}$, for paths starting at $\mathbf{i}_{0}$.) The quantity $p_{I}$ is an "irreducible propagator," which collects all terms in which the last "interaction" $\sigma$ occurs at time $t-1$ and in a bond ending in 
$\mathbf{i}_{t}$. We can write the "full" partition function in terms of these irreducible pieces as

$$
Z_{L}(\underline{\sigma})=\left\langle Z_{L}(\underline{\sigma})\right\rangle+\sum_{t=1}^{L} \sum_{\mathbf{i}_{t} \in \mathbf{Z}^{d}} p_{I}\left(t, \mathbf{i}_{t} ; 0\right) \text {. }
$$

Note that $\left\langle Z_{L}(\sigma)\right\rangle=1$. The idea of the proof of Proposition 3.7 is now to show that with large probability, $Z_{L}(\sigma)$ is close to its mean 1.

We choose a particularly convenient subsequence of $L$ 's in order to evaluate the bound in (3.16). Let $L_{0}=0$ and, for $j>0, L_{j}=2^{j}$. We will consider the partition functions $Z_{L_{j}}$ and we rewrite them as

$$
Z_{L_{m}}(\underline{\sigma})=1+\sum_{j=1}^{m} S\left(L_{j-1}, L_{j} ; 0\right)
$$

where we define, for $t_{2}>t_{1} \geqq s$,

$$
S\left(t_{1}, t_{2} ; s\right)=\sum_{t_{1}<t \leqq t_{2}} \sum_{\mathbf{i}_{t} \in \mathbf{Z}^{d}} p_{I}\left(t, \mathbf{i}_{t} ; s\right) .
$$

Note that for $s^{\prime}>0, S\left(t_{1}+s^{\prime}, t_{2}+s^{\prime} ; s+s^{\prime}\right)$ has the same distribution as $S\left(t_{1}, t_{2} ; s\right)$. We next state a basic bound on $S$ :

Proposition 3.8. If $\sigma^{2}$ is sufficiently small and $d \geqq 3$, there exists a constant $C_{1}>0$ such that for all non-negative $t^{*}, t_{1}<t_{2}$ one has

$$
\left\langle\left|S\left(t^{*}+t_{1}, t^{*}+t_{2} ; t^{*}\right)\right|^{2}\right\rangle \leqq C_{1} \sigma^{2}\left(1+t_{1}\right)^{-(d-2) / 2} .
$$

Corollary 3.9. Under the hypotheses of the previous proposition, there are constants $C_{2}$ and $C_{3}$ such that

for $0<\eta<1 / 2$.

$$
\begin{aligned}
& \operatorname{Prob}\left(\left\{\underline{\sigma}|| S\left(t^{*}+t_{1}, t^{*}+t_{2} ; t^{*}\right) \mid>\left(C_{2} \sigma^{2}\left(1+t_{1}\right)^{-(d-2) / 2}\right)^{\eta}\right\}\right) \\
& \quad \leqq C_{3} \sigma^{2(1-2 \eta)}\left(1+t_{1}\right)^{-(1-2 \eta)(d-2) / 2}
\end{aligned}
$$

Proof. This is an immediate consequence of Proposition 3.8 and of Chebyshev's inequality.

We now fix $\eta$ in $(0,1 / 2)$. Postponing the proof of Proposition 3.8 we continue the proof of Proposition 3.7. We consider first special noise configurations $\underline{\sigma}$.

Definition 3.10. We say a noise configuration $\underline{g}$ is eventually good if there is some $m^{*}=m^{*}(\sigma)<\infty$ such that

$$
\left|S\left(L_{j}, L_{j+1} ; L_{m^{*}}\right)\right|<\left(C_{2} \sigma^{2}\left(1+L_{j-m^{*}}\right)^{-(d-2) / 2}\right)^{\eta},
$$

for all $j \geqq m^{*}$.

Proposition 3.11. If $\sigma^{2}$ is sufficiently small, then for every $\sigma$ which is eventually good, one has

$$
\lim _{m \rightarrow \infty} \frac{1}{L_{m}} \log ^{-}\left(Z_{L_{m}}(\sigma)\right)=0 .
$$

We shall see below that almost all $\underline{\sigma}$ are eventually good.

Proof. Assume $\underline{\sigma}$ is eventually good and let $m^{*}$ be defined as above. Choose $L=L_{m}$, with $m>m^{*}$ and set $t^{*}=L_{m^{*}}$. We will bound $Z_{L}(\sigma)$ from below by again restricting 
the sum over random walks, much as we did in the proof of Proposition 3.2. With the notation of (3.5), (but now for $M=\infty$ ), we have

$$
\begin{aligned}
& Z_{L}(\sigma)=\sum_{\mathbf{i}: \mathbf{i}^{(0)}=\mathbf{i}_{0}} w_{L}(\underline{\mathbf{i}}) \prod_{p=0}^{L-1}(1+\sigma(p ; \underline{\mathbf{i}})) \\
& \geqq \sum_{\mathbf{i}: \mathbf{i}^{(0)}=\mathbf{i}^{\left(t^{*}\right)}=\mathbf{i}_{0}} w_{L}(\mathbf{i}) \prod_{p=0}^{L-1}(1+\sigma(p ; \mathbf{i}))
\end{aligned}
$$

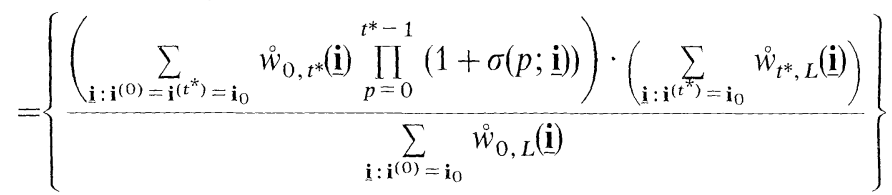

$$
\begin{aligned}
& \times\left\{\sum_{\mathbf{i}: \mathbf{i}^{\left(t^{*}\right)}=\mathbf{i}_{0}} w_{t^{*}, L}(\underline{\mathbf{i}}) \prod_{p=t^{*}}^{L-1}(1+\sigma(p ; \underline{\mathbf{i}}))\right\},
\end{aligned}
$$

where the last sum is over walks starting at time $t^{*}$ and ending at time $L$ and $w_{t^{*}, L}$ is the corresponding (normalized) weight cf. (2.8).

We now claim that both factors in braces are bounded from below by a constant which is independent of $L$. We shall denote the quantity in the second pair of braces by $Z\left(t^{*}, L\right)$. Note that in terms of this notation, $Z_{L}=Z(0, L)$. More generally, we set

$$
Z\left(t_{1}, t_{2}\right)=\sum_{\mathbf{i}: \mathbf{i}^{\left(t_{1}\right)}=\mathbf{i}_{0}} w_{t_{1}, t_{2}}(\mathbf{i}) \prod_{p=t_{1}}^{t_{2}-1}(1+\sigma(p ; \mathbf{i})) .
$$

We can write

$$
Z\left(t^{*}, L_{m}\right)=1+\sum_{j=m^{*}}^{m-1} S\left(L_{j}, L_{j+1} ; t^{*}\right) .
$$

Using the bounds (3.20) on $S$, we see that the sum over $j$ converges and we have

$$
Z\left(t^{*}, L_{m}\right) \geqq 1-C_{2}^{\eta} \sigma^{2 \eta}\left(1-2^{-\eta(d-2) / 2}\right)^{-1},
$$

for all $m>m^{*}$. This bounds the second factor.

To bound the first factor, we remark that the hypothesis H.3 on the random variables implies, for some $\mu>0$,

$$
\prod_{p=0}^{t^{*}-1}(1+\sigma(p ; \underline{\mathbf{i}})) \geqq e^{-\mu t^{*}}
$$

We also have

$$
\sum_{\mathbf{i}: \mathbf{i}^{(0)}=\mathbf{i}^{\left(t^{*}\right)}=\mathbf{i}_{0}}{\stackrel{\circ}{t^{*}}}_{(\mathbf{i})} \geqq C_{4}\left(t^{*}\right),
$$

provided at least one term in the above sum is positive. This is the case since there is at least one path connecting the 2 endpoints, which only involves non-zero factors $f_{\mid \mathbf{i}^{(p+1)}-\mathbf{i}^{(p) \mid}}$, cf. (2.7). Thus, we have only to bound

$$
\frac{\sum_{\mathbf{i}: \mathbf{i}^{\left(t^{*}\right)}=\mathbf{i}_{0}} \dot{W}_{t^{*}, L}(\sigma)}{\sum_{\mathbf{i}: \mathbf{i}^{(0)}=\mathbf{i}_{0}} \dot{w}_{0, L}(\sigma)}
$$


Recalling how the $\stackrel{i}{w}$ are defined, we see that this quotient is exactly

$$
\frac{\left\|\langle\Gamma\rangle^{L-t^{*}} e\right\|}{\left\|\langle\Gamma\rangle^{L} e\right\|} \geqq C_{5}\left(t^{*}\right),
$$

and this inequality holds for all $L$. (For large $s$, the vector $\langle\Gamma\rangle^{L} e$ is essentially colinear with the eigenvector corresponding to the largest eigenvalue of $\langle\Gamma\rangle$, and hence the quotient in fact approaches $e^{-\mu_{1} t^{*}}$.) Thus, the first factor in braces [in (3.21)] is bounded by

$$
C_{4}\left(t^{*}\right) C_{5}\left(t^{*}\right) e^{-\mu t^{*}}
$$

for all $L$.

Combining (3.22) with this, we get

$$
\lim _{m \rightarrow \infty} \frac{1}{L_{m}} \log ^{-}\left(Z_{L_{m}}(\underline{\sigma})\right)=0,
$$

whenever $\sigma^{2}$ is sufficiently small. This proves Proposition 3.11.

It remains to show that a.e. $\sigma$ is eventually good, and to give the proof of Proposition 3.8.

Proposition 3.12. If $\sigma^{2}$ is sufficiently small, then almost every $\sigma$ is eventually good.

Proof. We note that for a $\underline{\sigma}$ to fail to be eventually good, there is, for every $m^{*}$, a minimal $j=j\left(m^{*}, \sigma\right) \geqq m^{*}$ for which (3.20) fails. We can now construct a sequence of $j_{n}$ as follows: Set $j_{0}=0$. Then define $m_{n}=j_{n-1}+1$ and $j_{n}=j\left(m_{n}, \sigma\right)$, for $n=1, \ldots$. Define

$$
t_{n}=L_{m_{n}}=L_{j_{n-1}+1},
$$

where we recall that $L_{k}=2^{k}$. If $\underline{\sigma}$ fails to be eventually good, then, with the above choice of the $j$ 's we have

$$
\left|S\left(L_{j_{n}}, L_{j_{n}+1} ; t_{n}\right)\right| \geqq\left(C_{2} \sigma^{2}\left(1+L_{j_{n}-j_{n-1}}\right)^{-(d-2) / 2}\right)^{\eta},
$$

for $n=1,2, \ldots$ Given $j_{1}, \ldots, j_{N}$ we denote by $\underline{\sigma}_{\left\{j_{1}, \ldots, j_{N}\right\}}$ the set of $\underline{\sigma}$ for which the above construction yields the $j_{i}$ in question. Note that $j_{n}>j_{n-1}$. We now define $\widetilde{F}_{N}$ as the set

$$
\mathscr{F}_{N}=\bigcup_{j_{N}>\ldots>j_{1}>0} \underline{\sigma}_{\left\{j_{1}, \ldots, j_{N}\right\}} .
$$

Clearly, $\bigcap_{N>0} \mathscr{\mathscr { F }}_{N}$ contains all $\underline{\sigma}$ which are not eventually good. The condition (3.24) for a given $n$ only depends on $\sigma^{(p)}$ with $p=t_{n}, \ldots, L_{j_{n}+1}-1$. By construction, $t_{n+1}$ $=L_{j_{n}+1}>L_{j_{n}+1}-1$. This implies that these conditions are independent for different $n$. Therefore, using Corollary 3.9, we find

$$
\begin{aligned}
\operatorname{Prob}\left(\mathscr{F}_{N}\right) & \leqq \sum_{j_{N}>\ldots>j_{1}>0} \operatorname{Prob}\left(\underline{\sigma}_{\left\{j_{1}, \ldots, j_{N}\right\}}\right) \\
& \leqq \sum_{j_{N}>\ldots>j_{1}>0} \prod_{m=1}^{N}\left(C_{3} \sigma^{2}(1-2 \eta)\left(1+L_{j_{m}-j_{m-1}}\right)^{-(1-2 \eta)(d-2) / 2}\right) \\
& \leqq\left(C \sigma^{2(1-2 \eta)}\left(1-2^{-(1-2 \eta)(d-2) / 2}\right)^{-1}\right)^{N} .
\end{aligned}
$$

Since $N$ is arbitrary, this proves Proposition 3.12 . 
Proof of Proposition 3.7. Since Proposition 3.11 and Proposition 3.12 imply that

$$
\lim _{m \rightarrow \infty} \frac{1}{L_{m}} \log { }^{-}\left(Z_{L_{m}}(\sigma)\right)=0,
$$

for a.e. $\sigma$, one has, by (3.15) and the dominated convergence theorem,

$$
\lim _{m \rightarrow \infty} \frac{1}{L_{m}}\left\langle\log ^{-}\left(Z_{L_{m}}(\underline{\sigma})\right)\right\rangle=0
$$

which is Proposition 3.7.

Proof of Proposition 3.8. We begin by noting that by time-independence the estimate will be independent of the starting time $T^{*}$ so that it suffices to do the estimates for $T^{*}=0$. Using the definition (3.17) of the irreducible kernel, we see that

$$
\left\langle\left|S\left(T_{1}, T_{2} ; 0\right)\right|^{2}\right\rangle=\sum_{\substack{T_{1}<t_{1} \leqq T_{2} \\ T_{1}<t_{2} \leqq T_{2}}} \sum_{\mathbf{i}_{1}, \mathbf{i}_{2}}\left\langle p_{I}\left(t_{1}, \mathbf{i}_{1} ; 0\right) p_{I}\left(t_{2}, \mathbf{i}_{2} ; 0\right)\right\rangle .
$$

We now note that because of the time-independence of the $\sigma^{(p)}$ 's,

$$
\langle\sigma(t ; \underline{\mathbf{i}}) \sigma(s ; \underline{\mathbf{j}})\rangle=0,
$$

unless $t=s$, and the bonds $\mathbf{b}=\left(\mathbf{i}^{(t)}, \mathbf{i}^{(t+1)}\right)$ and $\mathbf{b}^{\prime}=\left(\mathbf{j}^{(s)}, \mathbf{j}^{(s+1)}\right)$ are neighbors in the sense that they either coincide or at least have a common endpoint. This remark allows us to expand (3.25), and to rewrite it, using (3.17), as

$$
\begin{aligned}
\left\langle\left|S\left(T_{1}, T_{2} ; 0\right)\right|^{2}\right\rangle= & \sum_{T_{1}<t \leq T_{2}} \sum_{n=1}^{T_{2}} \sum_{0=s_{0}<s_{1}<\ldots<s_{n}=t} \sum_{p=1} \sum_{\substack{\mathbf{j}^{\left(s_{p}-1\right)}, \mathbf{i}^{\left(s_{p}\right)} \\
\mathbf{j}^{\left(s_{p}-1\right)}, \mathbf{j}^{\left(s_{p}\right)}}}\left\langle\sigma\left(s_{p}-1 ; \underline{\mathbf{i}}\right) \sigma\left(s_{p}-1 ; \underline{\mathbf{j}}\right)\right\rangle \\
& \times P_{0}^{S_{p}-1-s_{p}-1}\left(\mathbf{i}^{\left(_{p}-1\right)}-\mathbf{i}^{\left(s_{p-1}\right)}\right) P_{0}^{s_{p}-1-s_{p-1}}\left(\mathbf{j}^{\left(s_{p}-1\right)}-\mathbf{j}^{\left(s_{p-1}\right)}\right) .
\end{aligned}
$$

The $s_{j}$ can be viewed as "hitting times" of the paths $\underline{\mathbf{i}}$ and $\underline{\mathbf{j}}$. Here, we take $\mathbf{i}^{(0)}=\mathbf{j}^{(0)}$ $=\mathbf{i}_{0}$. The probabilities $P_{0}^{t}$ are defined by

$$
P_{0}^{t}(\mathbf{j})=\sum_{\substack{\mathbf{i}: \mathbf{i}^{(0)}=\mathbf{i}_{0} \\ \mathbf{i}^{(t)}=\mathbf{j}}} w_{0, t}(\mathbf{i}) .
$$

The factors of $P_{0}$ result from the fact that in between their times of intersection, the walks $\underline{\mathbf{i}}$ and $\mathbf{j}$ are just "free" random walks on the infinite lattice in dimension $d$.

Note now that $\left|\mathbf{i}^{\left(s_{p}-1\right)}-\mathbf{j}^{\left(s_{p}-1\right)}\right|,\left|\mathbf{i}^{\left(s_{p}-1\right)}-\mathbf{i}^{\left(s_{p}\right)}\right|$, and $\left|\mathbf{j}^{\left(s_{p}-1\right)}-\mathbf{j}^{\left(s_{p}\right)}\right|$, must be less than or equal to 2 in order to get a non-zero contribution in the sum. This reduces the four-fold summations in (3.27) effectively to summation over a single index $\mathbf{i}$. On the other hand, for fixed $\mathbf{i}^{(u)}, \mathbf{j}^{(u)}$, we get using Corollary 5.3 of Sect. 5 for $\left|\mathbf{i}^{(u)}-\mathbf{j}^{(u)}\right| \leqq 2$,

$$
\sum_{\left|\mathbf{i}^{(v)}-\mathbf{j}^{(v)}\right| \leqq 2} P_{0}^{u-v}\left(\mathbf{i}^{(u)}-\mathbf{i}^{(v)}\right) P_{0}^{u-v}\left(\mathbf{j}^{(u)}-\mathbf{j}^{(v)}\right) \leqq \frac{C_{6}}{|u-v|^{d / 2}} .
$$

Furthermore, the factors of

$$
\left\langle\sigma\left(s_{p}-1 ; \underline{\mathbf{i}}\right) \sigma\left(s_{p}-1 ; \underline{\mathbf{j}}\right)\right\rangle
$$


are all bounded by $\sigma^{2}$. Summing over the $\mathbf{i}^{\left(s_{p}\right)}$ and $\mathbf{j}^{\left(s_{p}\right)}$ in (3.27) we find

$$
\begin{aligned}
\left\langle\left|S\left(T_{1}, T_{2} ; 0\right)\right|^{2}\right\rangle \leqq & C \sum_{T_{1}<t \leqq T_{2}} \sum_{n=1}^{T_{2}} \sigma^{2 n} \\
& \times \sum_{0=s_{0}<s_{1}<\ldots<s_{n}=t} C_{6}^{n} \prod_{p=1}^{n}\left(S_{p}-S_{p-1}\right)^{-d / 2} .
\end{aligned}
$$

Note that in the product, $\prod_{p=1}^{n}\left(s_{p}-s_{p-1}\right)^{-d / 2}$, there must be some $p$ with $s_{p}-s_{p-1}$ $>t / n$. (In all cases, $s_{p}-s_{p-1} \geqq 1$.) Thus

$$
\sum_{0=s_{0}<s_{1}<\ldots<s_{n}=t} \prod_{p=1}^{n}\left(s_{p}-s_{p-1}\right)^{-d / 2} \leqq C^{n} n\left(\frac{n}{t}\right)^{d / 2} .
$$

This implies

$$
\begin{aligned}
\left\langle\left|S\left(T_{1}, T_{2} ; 0\right)\right|^{2}\right\rangle & \leqq C \sum_{T_{1}<t \leqq T_{2}} \sum_{n=1}^{T_{2}} \frac{1}{t^{d / 2}}\left(C_{7} \sigma^{2}\right)^{n} n^{1+d / 2} \\
& \leqq C_{1} \sigma^{2}\left(1+T_{1}\right)^{-(d-2) / 2},
\end{aligned}
$$

for sufficiently small $\sigma^{2}$. This completes the proof of Proposition 3.8.

\section{Symplectic Matrices}

In the present section we demonstrate that the results of the previous sections generalize to the case of symplectic matrices

$$
\Sigma=\left(\begin{array}{cc}
1 & \Omega \\
1 & 1+\Omega
\end{array}\right)
$$

introduced in Sect. 1. Unlike the previous case of random Laplacians we cannot scale out the mean of the random variables $\omega$ in the symplectic case. We have to consider explicitly their mean and we define, throughout this section,

$$
\varepsilon=\left\langle\omega_{\mathbf{b}}\right\rangle \text {. }
$$

In analogy with our definition for the random Laplacian we define the random variables $\sigma_{\mathbf{x}^{(p)} \mathbf{x}^{(p+1)}}^{(p)}$ as in (2.9), by

$$
\sigma_{\mathbf{x y}}=\frac{\Sigma_{\mathbf{x y}}-\left\langle\Sigma_{\mathbf{x y}}\right\rangle}{\left\langle\Sigma_{\mathbf{x y}}\right\rangle},
$$

whenever $\left\langle\Sigma_{\mathbf{x y}}\right\rangle \neq 0$. We then set

$$
\sigma^{2}=\max _{\mathbf{b}}\left(\operatorname{var}\left(\sigma_{\mathbf{b}}\right)\right)
$$

In this section $\lambda_{M}$ refers to the largest Liapunov exponent of $\Sigma$, not $\Omega$.

\subsection{Statement of Results}

Our principal result is the following set of estimates on Liapunov exponents: 
Theorem 4.1. For all $d \geqq 1$ and all even $M$ one has the inequality

$$
\lambda_{M} \leqq \log \left(1+2 \varepsilon d+2 \sqrt{\varepsilon d+\varepsilon^{2} d^{2}}\right) .
$$

If $d \geqq 3$ and $\sigma^{2}$ is sufficiently small one has

$$
\lim _{M \rightarrow \infty} \lambda_{M}=\log \left(1+2 \varepsilon d+2 \sqrt{\varepsilon d+\varepsilon^{2} d^{2}}\right) .
$$

Remark. Note that in terms of the original random variables $\omega$, the condition $\sigma^{2}$ sufficiently small means that there exists a small constant $c>0$ such that $\operatorname{var}(\omega)$ $<c \varepsilon^{2}$.

Proof. We prove this theorem by comparing the Liapunov exponent, $\lambda_{M}$, to the (explicitly calculable) Liapunov exponents of the matrix averaged over the randomness. As a first step, we note that - for the purpose of computing Liapunov exponents - the argument we used in Sect. 2.3 allows us to replace the random matrices $\Omega$ by random matrices $\Gamma$, all of whose entries are non-negative. By an abuse of notation, we call the new matrices $\Sigma$ as well, and from now on we will consider only these latter matrices.

We next note that $\langle\Gamma\rangle=\varepsilon D_{M}^{+}$, where

$$
\left(D_{M}^{+}\right)_{\mathbf{i}, \mathbf{j}}= \begin{cases}1, & \text { if }|\mathbf{i}-\mathbf{j}|=1, \\ 2 d, & \text { if } \mathbf{i}=\mathbf{j}, \\ 0, & \text { otherwise }\end{cases}
$$

The subscript $M$ indicates that the distances are measured $\bmod M$. Thus, if we consider $\langle\Sigma\rangle$, we have

$$
\langle\Sigma\rangle=\left(\begin{array}{cc}
1 & \varepsilon D_{M}^{+} \\
1 & 1+\varepsilon D_{M}^{+}
\end{array}\right) .
$$

Let $\delta$ denote the largest eigenvalue of $D_{M}^{+}$. This eigenvalue is $4 d$. An elementary calculation shows then that the largest eigenvalue, $\mu$, of $\langle\Sigma\rangle$ is given by

$$
\mu=1+\frac{1}{2} \varepsilon \delta+\frac{1}{2} \sqrt{4 \varepsilon \delta+\varepsilon^{2} \delta^{2}} .
$$

Since $\mu$ is positive, the largest Liapunov exponent, $\bar{\lambda}_{M}$, of $\langle\Sigma\rangle$ is

$$
\bar{\lambda}_{M}=\log \left(1+2 \varepsilon d+2 \sqrt{\varepsilon d+\varepsilon^{2} d^{2}}\right) .
$$

Thus, Theorem 4.1 is equivalent to showing that in any dimension,

$$
\lambda_{M} \leqq \bar{\lambda}_{M}
$$

while for $d \geqq 3$ and $\sigma^{2}$ sufficiently small,

$$
\lim _{M \rightarrow \infty}\left(\lambda_{M}-\bar{\lambda}_{M}\right)=0
$$

Since (4.5) follows immediately from Proposition 2.4, we only need to establish (4.6), and Theorem 4.1 follows. This is again done via a random walk expansion.

\subsection{The Random Path Expansion for Symplectic Matrices}

One has a random path expansion for random products $\Sigma^{n}$, with $\Sigma$ as above, which is similar to the one developed in Sect. 2.4 for the product of random Laplacians. 
The main idea of our method consists in viewing the space of indices $1, \ldots, 2 M^{d}$ of the $2 M^{d} \times 2 M^{d}$ matrices $\Sigma$ as elements of a two layered torus. More precisely, we associate to every index $1 \leqq i \leqq 2 M^{d}$ a pair of indices $(j, \alpha)$ by defining a map $\phi$ : The indices of $\Sigma$ are naturally divided into two groups, corresponding to the representation (4.1) of $\Sigma$. We set

$$
\phi(i)= \begin{cases}(\mathbf{i}, u), & \text { if } \quad 1 \leqq i \leqq M^{d}, \\ (\mathbf{i}, \ell), & \text { if } \quad M^{d}+1 \leqq i \leqq 2 M^{d},\end{cases}
$$

where $\mathbf{i}$ is associated with the index in the corresponding submatrix, as in the case of random Laplacians. Thus the index set $1, \ldots, 2 M^{d}$ is identified with $\mathbf{I}_{M} \times \mathscr{L}$ where $\mathbf{I}_{M}$ is, as before, the torus of side $M$, and $\mathscr{L}$ is the set of "levels" which we call "upper" (case of $u$ ) and "lower" (case of $\ell$ ). Note that this periodicity is in agreement with periodicity of the matrices $\Omega$ or $\Gamma$ occurring in $\Sigma$. We now define weights in analogy with Sect. 2.4, corresponding to the averaged matrix $\langle\Sigma\rangle$. We set

$$
\begin{gathered}
f_{u u}(0)=1, \quad f_{\ell u}(0)=1, \quad f_{u \ell}(0)=\varepsilon 2 d, \\
f_{u \ell}(1)=\varepsilon, \quad f_{\ell \ell}(0)=1+\varepsilon 2 d, \quad f_{\ell \ell}(1)=1+\varepsilon .
\end{gathered}
$$

All other values of $f_{\alpha \beta}(n)$ are set equal to zero. We now assign to every walk $\underline{\mathbf{x}}$ of length $t$ in $\left(\mathbf{I}_{M} \times \mathscr{L}\right)^{t}$ the weight

$$
\grave{w}_{t}^{(M)}(\underline{\mathbf{x}})=\prod_{p=0}^{t-1} f_{\mathbf{x}_{2}^{(p+1)} \mathbf{x}_{2}^{(p)}}\left(\left|\mathbf{x}_{1}^{(p+1)}-\mathbf{x}_{1}^{(p)}\right|\right)
$$

where we use the projections onto components

$$
\mathbf{x}^{(p)}=\left(\pi_{1}\left(\mathbf{x}^{(p)}\right), \pi_{2}\left(\mathbf{x}^{(p)}\right)\right),
$$

i.e., the "1" component is in $\mathbf{I}_{M}$ and the " 2 " component in $\mathscr{L}$. Now let the vector $e \in \mathbf{R}^{\mathbf{I}_{M} \times \mathscr{L}}$ have a component $\mathbf{x}_{0}=\left(\mathbf{i}_{0}, \alpha_{0}\right)=1$ and all others zero. Define the norm on the space $\mathbf{R}^{\mathbf{I}_{M} \times \mathscr{L}}$ as the $L^{1}$ norm of the components. Then we have with the above notation:

$$
\left\|\langle\Sigma\rangle^{t} e\right\|=\sum_{\underline{\mathbf{x}}: \mathbf{x}^{(0)}=\mathbf{x}_{0}} \stackrel{\circ}{t}_{t}^{(M)}(\underline{\mathbf{x}})
$$

We again define

$$
\stackrel{\circ}{Z}_{L}^{(M)}(\underline{\sigma})=\sum_{\mathbf{x}: \mathbf{x}^{(0)}=\mathbf{x}_{0}} \dot{\leftrightarrow}_{t}^{(M)}(\underline{\mathbf{x}}) \prod_{p=0}^{t-1}\left(1+\sigma_{\mathbf{x}^{(p)} \mathbf{x}^{(p+1)}}^{(p)}\right),
$$

where the random variables $\sigma_{\mathbf{x}^{(p)}}^{(p)} \mathbf{x}^{(p+1)}$ are defined, as in (4.2). We also define the normalized partition function $Z_{L}$ by

$$
Z_{L}^{(M)}(\underline{\sigma})=\frac{\check{Z}_{L}^{(M)}(\underline{\sigma})}{\left\langle\dot{Z}_{L}^{(M)}(\underline{\sigma})\right\rangle}
$$

We need some more notation to take care of the two levels. We shall always fix the "position" $\mathbf{i}_{0}$ at which the paths start, but we will have to allow for both "levels" in the evaluation of $Z$. Thus, we define

$$
\stackrel{Z}{L}_{L}^{(M)}(\alpha, \underline{\sigma})=\sum_{\mathbf{x}: \mathbf{x}^{(0)}=\left(\mathbf{i}_{0}, \alpha\right)} \dot{w}_{t}^{(M)}(\underline{\mathbf{x}}) \prod_{p=0}^{t-1}\left(1+\sigma_{\mathbf{x}^{(p)} \mathbf{x}^{(p+1)}}^{(p)}\right) .
$$




\subsection{Reduction to the Infinite Volume Case}

We follow Sect. 3.1 quite closely, and remark only on the changes due to the more complicated state space. Our principal result is again

Proposition 4.2. There is a constant $C$ such that for any $L$, and for any dimension $d$, one has the inequality

$$
\frac{1}{\ell L}\left\langle\log \dot{Z}_{\ell L}(\underline{\sigma})\right\rangle \geqq \min _{\alpha \in \mathscr{L}} \frac{1}{L}\left\langle\log \stackrel{\circ}{L}_{L}(\alpha, \sigma)\right\rangle-\frac{C}{L} \log L,
$$

for $\ell=1,2, \ldots$ In particular,

$$
\lambda_{M}-\bar{\lambda}_{M} \geqq \frac{1}{L} \min _{\alpha \in \mathscr{L}}\left\langle\log Z_{L}^{(M)}(\alpha, \sigma)\right\rangle-\mathrm{C} \frac{\log M+\log L}{L} .
$$

Proof. Recall that

$$
\lambda_{M}=\lim _{t \rightarrow \infty} \frac{1}{t}\left\langle\log \stackrel{Z}{Z}_{t}^{(M)}(\underline{\sigma})\right\rangle .
$$

The proof is now an almost verbatim copy of the one for the random Laplacians, and is obtained by replacing the variable $\mathbf{i}$ in $\mathbf{I}_{M}$ by $\mathbf{x}$ in $\mathbf{I}_{M} \times \mathscr{L}$. The only new difficulty comes from the presence of the two "levels" and is connected with the difference in transition probabilities for these two levels. We now indicate where these things matter, by following the steps of the proof. The Definition 3.3 is unchanged (except for the replacement of $\mathbf{i}$ by $\mathbf{x}$ which we will not consider as a change any more). The argument goes through except for (3.7) which holds no longer. We now have that the distribution of $Z_{\mathbf{y}, \mathbf{y}_{j+1}(\underline{\sigma})}^{*}\left(t_{j}, t_{j+1} ; \sigma\right)$ (with $\mathbf{y}$, $\mathbf{y}_{j+1} \in \mathbf{I}_{M} \times \mathscr{L}$ ) can only take two values, depending on whether the second component of $\mathbf{y}$ is $u$ or $\ell$. (If the second components are fixed, then by the periodicity of the boundary conditions, the distribution is independent of the first component of $\mathbf{y}$.) Therefore, we find, instead of (3.8),

$$
\sum_{j=0}^{\ell-1}\left\langle\log Z_{\mathbf{y}_{j}(\underline{\sigma}), \mathbf{y}_{j+1}(\underline{\sigma})}^{*}\left(t_{j}, t_{j+1} ; \sigma\right)\right\rangle \geqq \ell \min _{\mathbf{y}_{0}=\left(\mathbf{i}_{0}, \alpha\right): \alpha \in \mathscr{L}}\left\langle\log Z_{\mathbf{y}_{0}, \mathbf{y}_{1}(\sigma)}^{*}(0, L ; \underline{\sigma})\right\rangle .
$$

It follows that (3.10) must be replaced by

$$
Z_{\mathbf{y}_{0}, \mathbf{y}_{1}(\sigma)}^{*}(0, L ; \sigma) \geqq \min _{\alpha \in \mathscr{L}} \frac{C}{L^{d}} \grave{Z}_{L}(\alpha, \sigma) .
$$

We see that (4.11) holds. We turn to the evaluation of $\left\langle Z_{t \ell}(\alpha, \underline{\sigma})\right\rangle$. Note again that

$$
\left\langle\dot{Z}_{t \ell}(\alpha, \sigma)\right\rangle=\left\|\langle\Sigma\rangle^{t_{\ell}} e_{\alpha}\right\|,
$$

where $e_{\alpha}$ is the vector in $\mathbf{R}^{\mathbf{I}_{M} \times \mathscr{L}}$ whose component $\left(\mathbf{i}_{0}, \alpha\right)$ equals 1 and all others are zero. Because neither $\left(\mathbf{i}_{0}, u\right)$ nor $\left(\mathbf{i}_{0}, \ell\right)$ are orthogonal to the eigenvector of the largest eigenvalue of $\langle\Sigma\rangle$, we find that

$$
\lim _{t \rightarrow \infty} \frac{1}{t} \log \left\|\langle\Sigma\rangle^{t} e_{\alpha}\right\|
$$


is independent of $\alpha$, and $\left\|\langle\Sigma\rangle^{t} e_{\alpha}\right\|=$ const $\mu_{1}^{t}\left(1+\mathcal{O}\left(M\left(\frac{\mu_{2}}{\mu_{1}}\right)^{t}\right)\right)$, where $\mu_{1}$ and $\mu_{2}$ are respectively the largest and second largest eigenvalues of $\langle\Sigma\rangle$. The analogue of Proposition 3.6 follows word for word from the proof in Sect. 3 and we find

Proposition 4.3. If $M$ is even and $L<M / 2$ and $\underline{\sigma}^{(M)}$ coincides with $\underline{\sigma}^{\prime(\infty)}$ in a disk of radius $L<M / 2$ around $\mathbf{i}_{0}$ one has

$$
Z_{L}^{(M)}\left(\alpha, \underline{\sigma}^{(M)}\right)=Z_{L}^{(\infty)}\left(\alpha, \underline{\sigma}^{(\infty)}\right) .
$$

for all $\alpha$.

Thus we may concentrate our attention on the infinite volume case. Again following the outline of Sect. 3 we see that the proof of Theorem 4.1 will follow if we prove

Proposition 4.4. If $\sigma^{2}$ is sufficiently small, and $d \geqq 3$, then we have, for $L_{j}=2^{j}$, and any $\alpha$,

$$
\lim _{j \rightarrow \infty} \frac{1}{L_{j}}\left\langle\log ^{-}\left(\mathrm{Z}_{L_{j}}(\alpha, \sigma)\right)\right\rangle=0 .
$$

The proof of this proposition is again very similar to the random Laplacian case. One first defines

$$
p_{I}\left(t, \mathbf{x}_{t} ; s\right)=\sum_{\mathbf{x}: \mathbf{x}^{(s)}=\mathbf{x}_{0}, \mathbf{x}^{(t)}=\mathbf{x}_{t}} \sigma(t-1 ; \underline{\mathbf{x}}) w_{s, t}(\underline{\mathbf{x}}) \prod_{p=s}^{t-2}(1+\sigma(p ; \underline{\mathbf{x}})),
$$

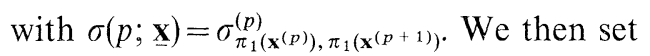

$$
S\left(t_{1}, t_{2} ; s\right)=\sum_{t_{1}<t \leqq t_{2}} \sum_{\mathbf{x}_{t} \in \mathbf{Z}^{d} \times \mathscr{L}} p_{I}\left(t, \mathbf{x}_{t} ; s\right) .
$$

Once again one can expand the partition function as

$$
Z_{L_{m}}(\underline{\sigma})=1+\sum_{j=1}^{m} S\left(L_{j-1}, L_{j} ; 0\right)
$$

and then one has the following two propositions:

Proposition 4.5. If $\sigma^{2}$ is sufficiently small and $d \geqq 3$, there exists a constant $C_{1}>0$ such that for all non-negative $t^{*}, t_{1}<t_{2}$ one has

$$
\left\langle\left|S\left(t^{*}+t_{1}, t^{*}+t_{2} ; t^{*}\right)\right|\right\rangle \leqq C_{1} \sigma^{2}\left(1+t_{1}\right)^{-(d-2) / 2} .
$$

Corollary 4.6. Under the hypotheses of the previous proposition, there are constants $\mathrm{C}_{2}$ and $\mathrm{C}_{3}$ such that

$$
\begin{aligned}
& \operatorname{Prob}\left(\left\{\underline{\sigma}|| S\left(t^{*}+t_{1}, t^{*}+t_{2} ; t^{*}\right) \mid<\left(C_{2} \sigma^{2}\left(1+t_{1}\right)^{-(d-2) / 2}\right)^{\eta}\right\}\right) \\
& \quad \leqq C_{3} \sigma^{2(1-2 \eta)}\left(1+t_{1}\right)^{-(1-2 \eta)(d-2) / 2},
\end{aligned}
$$

for $0<\eta<1 / 2$.

The proof of the corollary is immediate given the proposition. The proof of the proposition follows that of Proposition 3.8. 
Proof of Proposition 4.5. The time independence of the random variables allows us to take $T^{*}=0$. Then

$$
\left\langle\left|S\left(T_{1}, T_{2} ; 0\right)\right|^{2}\right\rangle=\sum_{\substack{T_{1}<t_{1} \leqq T_{2} \\ T_{1}<t_{2} \leqq T_{2}}} \sum_{\mathbf{i}_{1}, \mathbf{i}_{2}}\left\langle p_{I}\left(t_{1}, \mathbf{i}_{1} ; 0\right) p_{I}\left(t_{2}, \mathbf{i}_{2} ; 0\right)\right\rangle .
$$

We now note that because of the time-independence of the $\sigma^{(p)}$ 's,

$$
\langle\sigma(t ; \underline{\mathbf{x}}) \sigma(s ; \underline{\mathbf{y}})\rangle=0,
$$

unless $t=s$, and the bonds $\mathbf{b}=\left(\pi_{1}\left(\mathbf{x}^{(t)}\right), \pi_{1}\left(\mathbf{x}^{(t+1)}\right)\right)$ and $\mathbf{b}^{\prime}=\left(\pi_{1}\left(\mathbf{y}^{(s)}\right), \pi_{1}\left(\mathbf{y}^{(s+1)}\right)\right)$ are neighbors in the sense that they either coincide or at least have a common endpoint. This remark allows us to expand (4.20), and to rewrite it, using (4.17), as

$$
\begin{aligned}
\left\langle\left|S\left(T_{1}, T_{2} ; 0\right)\right|^{2}\right\rangle= & \sum_{T_{1}<t \leqq T_{2}} \sum_{n=1}^{T_{2}} \sum_{0=s_{0}<s_{1}<\ldots<s_{n}=t} \\
& \times \prod_{p=1}^{n} \sum_{\substack{\mathbf{x}^{\left(s_{p}-1\right)}, \mathbf{x}^{\left(s_{p}\right)} \\
\mathbf{y}^{\left(s_{p}-1\right)}, \mathbf{y}^{\left(s_{p}\right)}}}\left\langle\sigma\left(s_{p}-1 ; \mathbf{x}\right) \sigma\left(s_{p}-1 ; \underline{\mathbf{y}}\right)\right\rangle \\
& \times P_{\pi_{2}\left(\mathbf{x}^{\left(s_{p}-1\right)}, \pi_{2}\left(\mathbf{x}^{\left(s_{p}-1\right)}\right)\right.}^{s_{p}}\left(\pi_{1}\left(\mathbf{x}^{\left(s_{p}-1\right)}\right)-\pi_{1}\left(\mathbf{x}^{\left(s_{p}-1\right)}\right)\right) \\
& \times P_{\pi_{2}\left(\mathbf{y}^{\left(s_{p}-1\right)}, \pi_{2}\left(\mathbf{y}^{\left(s_{p}-1\right)}\right)\right.}^{s_{p}-1}\left(\pi_{1}\left(\mathbf{y}^{\left(s_{p}-1\right)}\right)-\pi_{1}\left(\mathbf{y}^{\left(s_{p}-1\right)}\right)\right),
\end{aligned}
$$

where we have set

$$
P_{\alpha, \beta}^{t}(\mathbf{i})=\frac{\sum^{*} \prod_{p=1}^{t} f_{\alpha_{p-1} \alpha_{p}}\left(\mathbf{i}^{(p-1)}-\mathbf{i}^{(p)}\right)}{\sum_{\substack{\mathbf{i}: \mathbf{i}^{(0)}=\mathbf{i}_{0}, \alpha_{0}, \ldots, \alpha_{t} \in \mathscr{L}: \alpha_{0}=\alpha}}^{t} \prod_{p=1}^{t} f_{\alpha_{p-1} \alpha_{p}}\left(\mathbf{i}^{(p-1)}-\mathbf{i}^{(p)}\right)},
$$

and used the notation

$$
\sum^{*}=\sum_{\substack{\mathbf{i}: \mathbf{i}^{(0)}=\mathbf{i}_{0}, \mathbf{i}^{(t)}=\mathbf{i} \\ \alpha_{0}, \ldots, \alpha_{t} \in \mathscr{L}: \alpha_{0}=\alpha, a_{t}=\beta}}
$$

From Corollary 5.3 we know that

$$
\sum_{\substack{\alpha, \beta \\ \alpha^{\prime}, \beta^{\prime}}} \sum_{\mathbf{i}^{(u)}-\mathbf{j}^{(u)} \mid \leqq 2} P_{\alpha, \beta}^{u-v}\left(\mathbf{i}^{(u)}-\mathbf{i}^{(v)}\right) P_{\alpha^{\prime}, \beta^{\prime}}^{u-v}\left(\mathbf{j}^{(u)}-\mathbf{j}^{(v)}\right) \leqq \frac{C}{|u-v|^{d / 2}}
$$

Inserting this estimate in (4.22) yields

$$
\left\langle\left|S\left(T_{1}, T_{2} ; 0\right)\right|^{2}\right\rangle \leqq C \sum_{T_{1}<t \leqq T_{2}} \sum_{n=1}^{T_{2}} \sigma^{2 n} \sum_{0=s_{0}<s_{1}<\ldots<s_{n}=t} C_{6}^{n} \prod_{p=1}^{n}\left(s_{p}-s_{p-1}\right)^{-d / 2},
$$

and from here the proof of Proposition 4.5 follows Sect. 3 word for word.

The proof of Theorem 4.1 is completed by defining the eventually good noise configurations in exactly the same fashion as in Sect. 3, namely,

Definition 4.7. We say a noise configuration $\sigma$ is eventually good if there is some $m^{*}=m^{*}(\underline{\sigma})<\infty$ such that 


$$
\left|S\left(L_{j}, L_{j+1} ; L_{m^{*}}\right)\right|<\left(C_{2} \sigma^{2}\left(1+L_{j-m^{*}}\right)^{-(d-2) / 2}\right)^{\eta},
$$

for all $j \geqq m^{*}$.

One then has

Proposition 4.8. If $\sigma^{2}$ is sufficiently small, then almost every $\underline{\sigma}$ is eventually good. and

Proposition 4.9. If $\sigma^{2}$ is sufficiently small, then for every $\sigma$ which is eventually good, one has

$$
\lim _{m \rightarrow \infty} \frac{1}{L_{m}} \log ^{-}\left(Z_{L_{m}}(\alpha, \sigma)\right)=0
$$

for all $\alpha$.

The proof of Proposition 4.8 follows verbatim from Sect. 3, while that of Proposition 4.9 involves only minor notational changes which we do not detail. These two propositions lead immediately to a proof of Proposition 4.4, and complete our discussion of the symplectic case.

\section{Random Walks in Three or More Dimensions}

In this section, we summarize some well known results about random walks in dimension 3 or more, and formulate results in the form in which we use them. The cases we consider are special cases of the more general setting of "aperiodic" random walks in the sense of Spitzer [10].

The state space in which our random walks take place is of one of two types:

1) The lattice $\mathscr{R}_{1} \equiv \mathbf{Z}^{\mathbf{d}}$, with $d \geqq 3$,

2) The "double-layered lattice" $\mathscr{R}_{2} \equiv \mathbf{Z}^{\mathbf{d}} \times \mathscr{L}$, where $\mathscr{L}$ is the set of "levels", $\mathscr{L}=\{u, \ell\}$, as we discussed it in Sect. 4.

The transition probabilities have already been defined in earlier sections. We summarize them here again.

1) In the case of the lattice Laplacian, cf. Eqs. (2.6)-(2.8) the transition probabilities are translation invariant. We get in the case of the infinitely extended domain, $M=\infty$, the transition probabilities

$$
P(\mathbf{i})=\frac{f_{|\mathbf{i}|}}{2^{d+1}} .
$$

We have used the notation from Sect. 2.4.

2) In the case of the symplectic matrices, we define $P_{\alpha \beta}(\mathbf{i})=f_{\alpha \beta}(\mathbf{i}) / \sum_{\mathbf{j}, \beta^{\prime}} f_{\alpha \beta^{\prime}}(\mathbf{j})$, cf. (4.8).

Note that we are looking here at the "free" walks, i.e., all $\omega_{\mathbf{i j}}^{(p)}$ are equal to 1 . In other words, they are defined in terms of the averaged matrices, $\langle\Omega\rangle$ and $\langle\Sigma\rangle$, and hence do not depend on the random variables $\underline{\sigma}$. 
We next state several facts to fix the terminology, although the proofs are obvious.

Lemma 5.1. The random walks, for $M=\infty$, defined by the Laplacians $\langle\Omega\rangle$ on $\mathscr{R}_{1}$ and by the symplectic matrices $\langle\Sigma\rangle$ on $\mathscr{R}_{2}$ can reach every point in their state space, starting at an arbitrary point in state space.

Proof. Assume a path starts at the "origin" [i.e., at $\mathbf{i}_{0}$ in $\mathscr{R}_{1}$ respectively at $\mathbf{i}_{0} \oplus \ell$ (or $\left.\left.\mathbf{i}_{0} \oplus u\right)\right]$ in $\mathscr{R}_{2}$. Given any other point $\mathbf{j}$ in $\mathscr{R}_{1}$, it is obvious that there is some finite power $m$ of the random matrices $\Omega(\omega)$ for which $\Omega^{m}(\underline{\omega})_{\mathbf{j}, \mathbf{i}_{0}}$ is not zero. The analogous statement holds in $\mathscr{R}_{2}$.

The above lemma implies that the random walks on $\mathscr{R}_{i}, i=1,2$ are aperiodic in the sense of [10].

We now discuss the case of walks in $\mathscr{R}_{2}$ only, the walks in $\mathscr{R}_{1}$ being an easier subcase. We define $P_{\alpha, \beta}^{t}(\mathbf{i})$ as the transition probability from $(\boldsymbol{0}, \alpha)$ to $(\mathbf{i}, \beta)$ in time $t$, $t>0$, and $F_{\alpha, \beta}^{t}(\mathbf{i})$ as the corresponding unnormalized weight, defined by

$$
F_{\alpha, \beta}^{t}(\mathbf{i})=\sum_{\substack{\mathbf{i}: \mathbf{i}^{(0)}=0, \mathbf{i}^{(t)}=\mathbf{i} \\ \alpha_{0}, \ldots, \alpha_{t} \in \mathscr{L}: \alpha_{0}=\alpha, \alpha_{t}=\beta}} \prod_{p=1}^{t} f_{\alpha_{p-1} \alpha_{p}}\left(\mathbf{i}^{(p-1)}-\mathbf{i}^{(p)}\right) .
$$

In terms of this definition, Lemma 5.1 is in fact proved by showing that for $t=2$ and arbitrary $\alpha$, one has

$$
F_{\alpha, \beta}^{t}(\mathbf{i})>0
$$

for $d$ linearly independent directions in $\mathbf{Z}^{d}$.

Proposition 5.2. If the conclusions of Lemma 5.1. hold, and if $f_{\alpha \beta}(\mathbf{i}) \neq 0$ for finitely many $\mathbf{i}$, then there is a constant $C$ such that for all $t>0$ one has

$$
\sum_{\mathbf{i} \in \mathbf{Z}^{d}}\left|\sum_{\beta \in \mathscr{L}} P_{\alpha, \beta}^{t}(\mathbf{i})\right|^{2} \leqq \frac{C}{t^{d / 2}} .
$$

Proof. This proposition is a slight generalization of results in Spitzer. We first define the quadratic forms $Q_{\alpha, \beta}$, for $\alpha, \beta \in \mathscr{L}$, by

$$
Q_{\alpha, \beta}(\theta)=\sum_{\mathbf{i} \in \mathbf{Z}^{d}}(\theta \cdot \mathbf{i})^{2} F_{\alpha, \beta}^{2}(\mathbf{i}) .
$$

Note that because $F_{\alpha, \beta}^{t=1}(\mathbf{i})$ is zero when $|\mathbf{i}|>1$, the sum in (5.2) extends only over $|\mathbf{i}| \leqq L \equiv 2$. The forms $Q_{\alpha, \beta}$ are positive definite by (5.1). Therefore, there is a $\lambda>0$ such that

$$
Q_{\alpha, \beta}(\theta) \geqq \lambda(\theta \cdot \theta),
$$

for all $\theta \in \mathbf{R}^{d}$. We next define the characteristic functions $\Phi_{\alpha \beta}$ by

$$
\Phi_{\alpha \beta}(\theta)=\sum_{\mathbf{i} \in \mathbf{Z}^{d}} F_{\alpha, \beta}^{2}(\mathbf{i}) e^{i \theta \cdot \mathbf{i}} .
$$

Since our random walks are symmetric, i.e., $F_{\alpha, \beta}^{t}(\mathbf{i})=F_{\alpha, \beta}^{t}(-\mathbf{i})$, we see that $\Phi_{\alpha, \beta}$ is real, and we have the identity

$$
\Phi_{\alpha \beta}(0)-\Phi_{\alpha \beta}(\theta)=\sum_{\mathbf{i} \in \mathbf{Z}^{d}}(1-\cos (\theta \cdot \mathbf{i})) F_{\alpha, \beta}^{2}(\mathbf{i})=2 \sum_{\mathbf{i} \in \mathbf{Z}^{d}} \sin ^{2}\left(\frac{\theta \cdot \mathbf{i}}{2}\right) F_{\alpha, \beta}^{2}(\mathbf{i}) .
$$


When $|\theta|<\pi / L$, we have $\sin ^{2}(\theta \cdot \mathbf{i} / 2) \geqq(\theta \cdot \mathbf{i} / \pi)^{2}$ in the above sum because of the support properties of $F_{\alpha, \beta}^{2}$. When $|\theta| \geqq \pi / L$ and $\theta \in \mathscr{C} \equiv[-\pi, \pi]^{d}$, we have $\Phi_{\alpha \beta}(0)$ $-\Phi_{\alpha \beta}(\theta)>0$, since $\Phi_{\alpha \beta}(0)=\Phi_{\alpha \beta}(\theta)$ in $\mathscr{C}$ only if $\theta=0$. Thus, for some $\lambda^{\prime}>0$, we have

$$
\Phi_{\alpha \beta}(0)-\Phi_{\alpha \beta}(\theta) \geqq \lambda^{\prime}(\theta \cdot \theta) \text {, }
$$

for $\theta \in \mathscr{C}$. In other words,

$$
0 \leqq \Phi_{\alpha \beta}(\theta) \leqq \Phi_{\alpha \beta}(0) e^{-\lambda^{\prime \prime}(\theta \cdot \theta)} .
$$

Note next that

$$
\sum_{\mathbf{i} \in \mathbf{Z}^{d}}\left|F_{\alpha_{0} \beta}^{2 t}(\mathbf{i})\right|^{2} \leqq \sum_{\mathbf{i} \in \mathbf{Z}^{d}}\left|\sum_{\alpha_{t} \in \mathscr{L}} F_{\alpha_{0} \alpha_{t}}^{2 t}(\mathbf{i})\right|^{2}
$$

On the other hand,

$$
\sum_{\alpha_{t} \in \mathscr{L}} F_{\alpha_{0} \alpha_{t}}^{2 t}(\mathbf{i})=\frac{1}{(2 \pi)^{d}} \int_{\mathscr{C}} d \theta e^{-i \theta \cdot \mathbf{i}} \sum_{\alpha_{1}, \ldots, \alpha_{t} \in \mathscr{L}} \prod_{p=1}^{t} \Phi_{\alpha_{p-1} \alpha_{p}}(\theta) .
$$

Combining (5.4) and (5.5), we see that

$$
\begin{aligned}
\sum_{\mathbf{i} \in \mathbf{Z}^{d}}\left|F_{\alpha_{0} \beta}^{2 t}(\mathbf{i})\right|^{2} & \leqq \frac{1}{(2 \pi)^{d}} \int_{\mathscr{C}} d \theta \sum_{\alpha_{1}, \ldots, \alpha_{t} \in \mathscr{L}} \prod_{p=1}^{t} \Phi_{\alpha_{p-1} \alpha_{p}}(\theta) \times \sum_{\alpha_{1}^{\prime}, \ldots, \alpha_{t}^{\prime} \in \mathscr{L}} \prod_{p=1}^{t} \Phi_{\alpha_{p-1}^{\prime} \alpha_{p}^{\prime}}(\theta) \\
& \leqq \frac{1}{(2 \pi)^{d}} \int_{\mathscr{C}} d \theta\left(\sum_{\alpha_{1}, \ldots, \alpha_{t} \in \mathscr{L}} \prod_{p=1}^{t} \Phi_{\alpha_{p-1} \alpha_{p}}(0)\right)^{2} e^{-\lambda^{\prime \prime} t(\theta \cdot \theta)}
\end{aligned}
$$

where the second inequality used (5.3). We now note that with $\alpha_{t}=\beta$, one has

$$
\sum_{\alpha_{1}, \ldots, \alpha_{t}-1 \in \mathscr{L}} \prod_{p=1}^{t} \Phi_{\alpha_{p}-1 \alpha_{p}}(0)=\sum_{\mathbf{i} \in \mathbf{Z}^{d}} F_{\alpha, \beta}^{2 t}(\mathbf{i}),
$$

so that

$$
P_{\alpha_{0} \beta}^{2 t}(\mathbf{i})=\frac{F_{\alpha_{0} \beta}^{2 t}(\mathbf{i})}{\sum_{\alpha_{1}, \ldots, \alpha_{t-1} \in \mathscr{L}} \prod_{p=1}^{t} \Phi_{\alpha_{p-1} \alpha_{p}}(0)} .
$$

Combining this remark with (5.6), and then performing the Gaussian integral yields the claim of Proposition 5.2, for all even $t>0$. Since there are finitely many transitions per unit time, the assertion then follows for all $t>0$.

Corollary 5.3. For every finite $m$, there is a constant $C$ such that for all $t>0$ and all $\alpha, \beta, \alpha^{\prime}, \beta^{\prime}$ we have

$$
\sum_{\mathbf{i}, \mathbf{j}: \mathbf{i}=\mathbf{j} \mid \leqq m} P_{\alpha, \beta}^{t}(\mathbf{i}) P_{\alpha^{\prime} \beta^{\prime}}^{t}(\mathbf{j}) \leqq \frac{C}{t^{d / 2}} .
$$

Proof. We start by writing

$$
F_{\alpha, \beta}^{2 t}(\mathbf{i})=\frac{1}{(2 \pi)^{d}} \int_{\mathscr{C}} d \theta e^{-i \theta \cdot \mathbf{i}} \Phi_{\alpha \beta}^{t}(\theta) .
$$


Then

$$
\begin{aligned}
\sum_{\mathbf{i}, \mathbf{j}:|\mathbf{i}=\mathbf{j}| \leqq m} F_{\alpha, \beta}^{2 t}(\mathbf{i}) F_{\alpha^{\prime} \beta^{\prime}}^{2 t}(\mathbf{j}) & =\left|\sum_{\mathbf{i}, \mathbf{j}:|\mathbf{i}-\mathbf{j}| \leqq 1} \frac{1}{(2 \pi)^{2 d}} \int d \theta d \theta^{\prime} \Phi_{\alpha \beta}^{t}(\theta) \Phi_{\alpha^{\prime} \beta^{\prime}}^{t}\left(\theta^{\prime}\right) e^{i\left(\theta \mathbf{i}-\theta^{\prime} \mathbf{j}\right)}\right| \\
& \leqq \sum_{\mathbf{u}}\left|\sum_{\mathbf{i}} \frac{1}{(2 \pi)^{2 d}} \int d \theta d \theta^{\prime} \Phi_{\alpha \beta}^{t}(\theta) \Phi_{\alpha^{\prime} \beta^{\prime}}^{t}\left(\theta^{\prime}\right) e^{i\left(\theta-\theta^{\prime}\right) \cdot \mathbf{i}} e^{i \theta^{\prime} \cdot \mathbf{u}}\right| \\
& =\sum_{\mathbf{u}}\left|\frac{1}{(2 \pi)^{d}} \int d \theta d \theta^{\prime} \Phi_{\alpha \beta}^{t}(\theta) \Phi_{\alpha^{\prime} \beta^{\prime}}^{t}(\theta) e^{i \theta \cdot \mathbf{u}}\right| \\
& =\sum_{\mathbf{u}}\left|\frac{1}{(2 \pi)^{d}} \int d \theta d \theta^{\prime} \Phi_{\alpha \beta}^{t}(\theta) \Phi_{\alpha^{\prime} \beta^{\prime}}^{t}(\theta) e^{i \theta \cdot \mathbf{u}}\right| \\
& <C \frac{\Phi_{\alpha \beta}^{t}(0) \Phi_{\alpha^{\prime} \beta^{\prime}}^{t}(0)}{t^{d / 2}} .
\end{aligned}
$$

Here, the sum over $\mathbf{u}$ extends over the set of possible paths of length at most $m$ which can be taken from a given site. By definition, this set is finite for finite $m$. The claim of the corollary then follows by dividing by the normalizing factors.

\section{Numerical Experiments for $d=1$}

We have seen in Sect. 3 - for random Laplacians - that in dimension three or higher, the largest Liapunov exponent $\lambda_{M}$ is "annealed", i.e., it is equal to the Liapunov exponent $\bar{\lambda}_{M}$ of the product of the averaged matrices $\langle\Omega\rangle$, when $M \rightarrow \infty$. In this section we present numerical evidence that the self-averaging does not take place in dimension $d=1$. We have performed numerical experiments with the matrices $\Gamma$, as defined in Sect. 1 for a random variable $\sigma$ distributed uniformly in $[1-a, 1+a]$, where $a<1$, and for several values of the matrix size $M$.

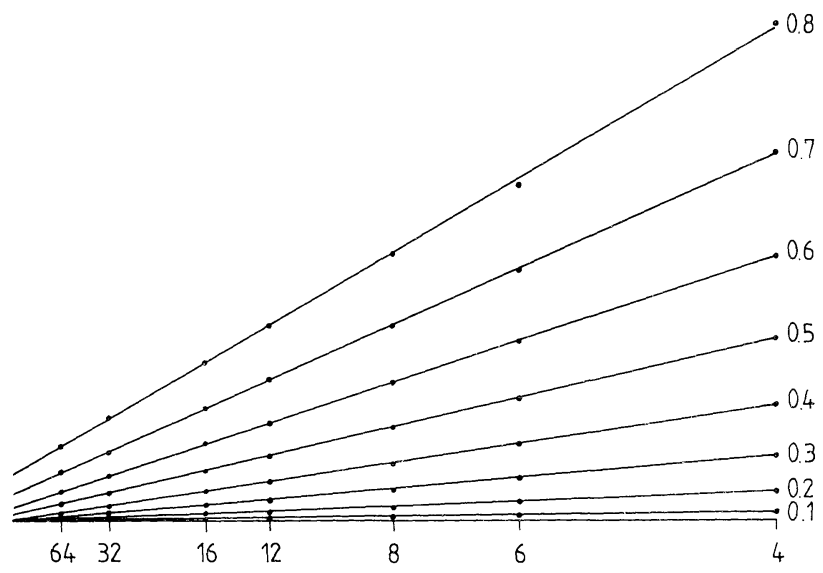

Fig. 1. Results of a numerical simulation. We show for various values of $M$ (horizontal axis), and various values of $a$ (labels on curve) the computed Liapunov exponents and the "theoretical fit". The vertical scale is proportional to $\bar{\lambda}_{M}-\hat{\lambda}_{M}$ 
In first approximation, we find a law

$$
\bar{\lambda}_{M}-\lambda_{M}(a) \sim C_{1} a^{2} / M+C_{2}(a),
$$

where $C_{1} \sim 0.1702$ and $C_{2}(a) \sim 0.0071 a^{4}$. The numerical evidence is summarized in Fig. 1, which shows that $C_{1}$ is reliably constant over the domain of $a$ considered, but that the fourth order behavior of $C_{2}$ is less clear. On the other hand, there seems to be some consistency with bounds which can be obtained from estimating, through the diffusion kernel, the number of intersections between different paths.

Acknowledgements. This work was partially supported by the Fonds National Suisse (Département de Physique Théorique, Genève, and Zentrum für Theoretische Studien, Zürich) and by the NSF, Grant DMS-8802118. CEW thanks the Département de Physique Théorique for its hospitality.

\section{References}

1. Bouchard, J.P., Georges, A., Hansel, D., Le Doussal, P., Maillard, J.M.: J. Phys. A 19, L 1452-L 1152 (1986)

2. Cohen, J.E., Newman, C.M.: The stability of large random matrices and their products. Ann. Prob. 12, 283-310 (1984)

3. Eckmann, J.-P., Wayne, C.E.: Liapunov spectra for infinite chains of non-linear oscillators. J. Stat. Phys. 50, 853-878 (1987)

4. Imbrie, J.Z., Spencer, T.: Directed polymers in a random environment (preprint)

5. Livi, R., Politi, A., Ruffo, S.: Distribution of characteristic exponents in the thermodynamic limit. J. Phys. A 19, 2033-2040 (1986)

6. Newman, C.M.: The distribution of Liapunov exponents. Commun. Math. Phys. 103, 121-126 (1986)

7. Newman, C.M.: Liapunov exponents for some products of random matrices: exact expressions and asymptotic distributions. In: Random matrices and their applications,"Vol. 50, p. 121. Cohen, J.E., Kesten, H., Newman, C.M. (eds.). Providence, RI: AMS Contemporary Mathematics 1986

8. Paladin, G., Vulpiani, A.: Scaling law and asymptotic distribution of Liapunov exponents in conservative dynamical systems with many degrees of freedom. J. Phys. A 19, 1881-1888 (1986)

9. Ruelle, D.: Ergodic theory of differentiable dynamical systems. Publ. IHES 50, 275-306 (1979)

10. Spitzer, F.: Principles of random walk, 2nd edn. Berlin, Heidelberg, New York: Springer 1976

Communicated by A. Jaffe

Received September 16, 1988 
